PREPARED FOR SUBMISSION TO JHEP

\title{
On the classical equivalence of monodromy matrices in squashed sigma model
}

\author{
lo Kawaguchi*1, Takuya Matsumoto ${ }^{\dagger, \ddagger 2}$ and Kentaroh Yoshida*3 \\ * Department of Physics, Kyoto University Kyoto 606-8502, Japan \\ $\dagger$ School of Mathematics and Statistics, University of Sydney, NSW 2006, Australia \\ $\ddagger$ Graduate School of Mathematics, Nagoya University, Nagoya 464-8602, Japan
}

\begin{abstract}
We proceed to study the hybrid integrable structure in two-dimensional non-linear sigma models with target space three-dimensional squashed spheres. A quantum affine algebra and a pair of Yangian algebras are realized in the sigma models and, according to them, there are two descriptions to describe the classical dynamics 1) the trigonometric description and 2) the rational description, respectively. For every description, a Lax pair is constructed and the associated monodromy matrix is also constructed. In this paper we show the gauge-equivalence of the monodromy matrices in the trigonometric and rational description under a certain relation between spectral parameters and the rescalings of $s l(2)$ generators.
\end{abstract}

Keywords: Integrable Field Theory, Sigma Models, AdS-CFT Correspondence

ArXiv EPrint: 1203.3400[hep-th]

\footnotetext{
${ }^{1}$ E-mail: io@gauge.scphys.kyoto-u.ac.jp

${ }^{2}$ E-mail: tmatsumoto@usyd.edu.au

${ }^{3}$ E-mail: kyoshida@gauge.scphys.kyoto-u.ac.jp
} 


\section{Contents}

1 Introduction $\quad 1$

2 Preliminaries $\quad 3$

2.1 The classical action of squashed sigma models 3

2.2 Trigonometric description 4

2.3 Rational description 8

3 The trigonometric/rational correspondence 10

3.1 Expansions of $U^{R}\left(\lambda_{R}\right) \quad 11$

3.2 Expansions of $U^{L_{ \pm}}\left(\lambda_{L_{ \pm}}\right) \quad 12$

$\begin{array}{lll}3.3 & \text { The relation of spectral parameters } & 15\end{array}$

3.4 The expansions of $U^{L_{ \pm}}\left(\lambda_{L_{ \pm}}\right)$: revisited 17

4 Gauge equivalence of monodromy matrices $\quad 19$

4.1 Gauge equivalence of monodromy matrices: $C=0 \quad 19$

4.2 Gauge equivalence of monodromy matrices: $C \neq 0 \quad 22$

4.3 Reduced trigonometric description and integrability 25

5 Conclusion and discussion $\quad 27$

\section{Introduction}

It is no exaggeration to say that the most fascinating topic in string theory is the AdS/CFT correspondence [1]. It provides a specific approach to quantum gravity as well as a useful tool to study strongly-coupled systems. An enormous amount of evidence support the duality, but still there is no rigorous proof of it. One may attempt to ask what mechanism is responsible for AdS/CFT. At the present time, the integrability is recognized as the fundamental structure of AdS/CFT (For a comprehensive review, see $[2])$.

The next issue is to consider integrable deformations of AdS/CFT. In this direction there are preceding works such as $\beta$-deformation [3] and its gravity dual [4-6], and

$q$-deformation of the world-sheet S-matrix [7-9]. Apart from them, we are interested in three-dimensional squashed spheres and warped $\mathrm{AdS}_{3}$. These geometries appear 
in recent studies like holographic condensed matter [10], Kerr/CFT [11] and warped $\mathrm{AdS}_{3} /$ dipole $\mathrm{CFT}_{2}[12,13]$. The potential applications to these topics make it significant to study the integrable structure of two-dimensional non-linear sigma models with target space warped $\mathrm{AdS}_{3}$ and squashed spheres.

In this paper we concentrate on the classical integrable structure of sigma models with squashed spheres. The reason is that warped $\mathrm{AdS}_{3}$ geometries are obtained via double Wick rotations of squashed spheres and the classical analysis performed here is valid irrespective of compactness of target space. We refer to the sigma models as "the squashed sigma models" as an abbreviation hereafter.

In a series of works [14-17] (For a short summary see [18]), we have shown that quantum affine algebra and Yangian algebra are realized in the squashed sigma models ${ }^{1}$. According to them, there are two descriptions to describe the classical dynamics: 1) the rational description and 2) the trigonometric description. Depending on the description, two kinds of Lax pair, which lead to the identical classical equations of motion, are constructed and also there are the corresponding monodromy matrices.

This means the "local" equivalence of the two descriptions and does not imply the equivalence of classical moduli spaces, namely "global" equivalence. In other words, the "global" equivalence is equivalent to the left-right symmetry. In fact, the "local" equivalence has been well known, while it has been believed that the "global" equivalence is not realized because the universality class of Lax pairs (i.e., topology of classical moduli space), spectral parameters and the number of poles are different between the two descriptions ${ }^{2}$.

We proceed here to study the classical integrable structure of squashed sigma models. We show the gauge-equivalence of monodromy matrices in the trigonometric and rational descriptions under the relation of spectral parameters and the rescalings of $s l(2)$ generators. As a result, the trigonometric description is shown to be equivalent to a composite of the rational descriptions. That is, the "global" equivalence is accurately realized even after squashing the target space geometry, in contrast to the folklore which has been believed so far without concrete proof. All of the difficulties mentioned in the previous paragraph are resolved by taking account of the two rational descriptions and finding out the relation between spectral parameters. Moreover, we find the "reduced" trigonometric description that works as the Lax pair at least at the classical level. With this description, the equivalence of the monodromy matrices becomes very apparent.

\footnotetext{
${ }^{1}$ The classical integrability is discussed also from T-duality argument [19].

${ }^{2}$ For example, see the sentence just below (2.18) of [20]. We are grateful to Adam Rej for drawing our attention to this article.
} 
This paper is organized as follows. In section 2 we introduce the squashed sigma models and the monodromy matrices in the trigonometric and rational descriptions. In section 3 the monodromy matrices are expanded around some points and the relation of spectral parameters is deduced. In section 4 we show the gauge-equivalence of monodromy matrices under the spectral parameter relation and the rescalings of $\operatorname{sl}(2)$ generators. The reducibility of the trigonometric Lax pair is also discussed. Section 5 is devoted to conclusion and discussion.

\section{Preliminaries}

We introduce the classical action of squashed sigma models and give a short review on a series of works [14-18], including some new results. Two descriptions to describe the classical dynamics are explained with monodromy matrices, which will be the main objects in the following discussion.

\subsection{The classical action of squashed sigma models}

First of all, let us introduce the $s u(2)$ Lie algebra generators $T^{a}(a=1,2,3)$ satisfying

$$
\left[T^{a}, T^{b}\right]=\varepsilon^{a b}{ }_{c} T^{c}, \quad \operatorname{Tr}\left(T^{a} T^{b}\right)=-\frac{1}{2} \delta^{a b} .
$$

The totally antisymmetric tensor $\varepsilon^{a b}{ }_{c}$ is normalized as $\varepsilon_{123}=+1$.

By using the left-invariant one-form,

$$
J \equiv g^{-1} d g, \quad g \in S U(2)
$$

the metric of squashed spheres in three dimensions is given by

$$
d s^{2}=-\frac{L^{2}}{2}\left[\operatorname{Tr}\left(J^{2}\right)-2 C\left(\operatorname{Tr}\left[T^{3} J\right]\right)^{2}\right] .
$$

The deformation parameter $C$ is a real constant supposed to be $C>-1$. When $C=0$, the metric (2.1) is reduced to that of round $S^{3}$ with radius $L$.

For $C \neq 0$, the $S^{3}$ isometry $S O(4)=S U(2)_{\mathrm{L}} \times S U(2)_{\mathrm{R}}$ is broken to $S U(2)_{\mathrm{L}} \times U(1)_{\mathrm{R}}$. The $S U(2)_{\mathrm{L}}$ transformation is just the left action and $U(1)_{\mathrm{R}}$ transformation is the right action generated by $T^{3}$,

$$
g \rightarrow g^{L} \cdot g \cdot \mathrm{e}^{-T^{3} \theta}
$$

The infinitesimal forms are

$$
\delta^{L, a} g=\epsilon T^{a} g, \quad \delta^{R, 3} g=-\epsilon g T^{3} .
$$


The minus sign in the right transformation law comes from the convention in (2.2) .

The classical action of squashed sigma models is given by

$$
S=\int_{-\infty}^{\infty} d t \int_{-\infty}^{\infty} d x \eta^{\mu \nu}\left[\operatorname{Tr}\left(J_{\mu} J_{\nu}\right)-2 C \operatorname{Tr}\left(T^{3} J_{\mu}\right) \operatorname{Tr}\left(T^{3} J_{\nu}\right)\right]
$$

where $x^{\mu}=(t, x)$ with the Lorentzian metric $\eta_{\mu \nu}=\operatorname{diag}(-1,1)$. We impose the boundary condition that $J_{\mu}$ vanishes at the spatial infinity. That is, the group field variable $g(t, x)$ approaches a constant element like ${ }^{3}$

$$
g(t, x) \rightarrow g_{\infty} \quad(x \rightarrow \pm \infty)
$$

The Virasoro constraints are not taken into account, for simplicity.

The classical equations of motion are

$$
\partial^{\mu} J_{\mu}-2 C \operatorname{Tr}\left(T^{3} \partial^{\mu} J_{\mu}\right) T^{3}-2 C \operatorname{Tr}\left(T^{3} J_{\mu}\right)\left[J^{\mu}, T^{3}\right]=0 .
$$

In the squashed sigma models, two infinite-dimensional symmetries 1) quantum affine algebra and 2) Yangian algebra are realized and hence two kinds of Lax pairs can be constructed depending on the symmetries. That is, there are two descriptions to describe the classical dynamics. We shall give a short summary of the two descriptions in the coming two subsections.

\subsection{Trigonometric description}

The one is the trigonometric description related to quantum affine algebra [17].

With the spectral parameter $\lambda_{R}$, the associated Lax pair is given by $[22]^{4}$

$$
\begin{aligned}
& L_{t}^{R}\left(x ; \lambda_{R}\right)=-\frac{1}{2} \sum_{a=1}^{3}\left[w_{a}\left(\alpha+\lambda_{R}\right) J_{+}^{a}+w_{a}\left(\alpha-\lambda_{R}\right) J_{-}^{a}\right] T^{a}, \\
& L_{x}^{R}\left(x ; \lambda_{R}\right)=-\frac{1}{2} \sum_{a=1}^{3}\left[w_{a}\left(\alpha+\lambda_{R}\right) J_{+}^{a}-w_{a}\left(\alpha-\lambda_{R}\right) J_{-}^{a}\right] T^{a},
\end{aligned}
$$

where the following quantities have been introduced,

$$
x^{ \pm} \equiv \frac{1}{2}(t \pm x), \quad J_{ \pm} \equiv J_{t} \pm J_{x}, \quad J_{\mu}^{a} \equiv-2 \operatorname{Tr}\left(T^{a} J_{\mu}\right) .
$$

\footnotetext{
${ }^{3}$ Seemingly, two independent, constant elements are allowed at the two endpoints $x= \pm \infty$. However, they must be identical by the gauge invariance of the trace of monodromy matrix.

${ }^{4}$ The study of squashed sigma models has a long history and the trigonometric Lax pair was originally constructed by Cherednik [21]. We here use the expression of the Lax pair in [22].
} 


$$
w_{1}\left(\lambda_{R}\right)=w_{2}\left(\lambda_{R}\right) \equiv \frac{\sinh \alpha}{\sinh \lambda_{R}}, \quad w_{3}\left(\lambda_{R}\right) \equiv \frac{\tanh \alpha}{\tanh \lambda_{R}}
$$

The parameter $\alpha$ is related to the squashing parameter $C$ as

$$
i \sqrt{C}=\tanh \alpha \text {. }
$$

Due to the relation (2.7) and the reality of $C, \alpha$ must be pure imaginary for $C>0$ and real, up to $i \pi n(n \in \mathbb{Z})$, for $-1<C<0$. Note that the value of $C$ is automatically restricted to the physical region $C>-1$. The following zero curvature condition

$$
\left[\partial_{t}-L_{t}^{R}\left(x ; \lambda_{R}\right), \partial_{x}-L_{x}^{R}\left(x ; \lambda_{R}\right)\right]=0
$$

leads the equations of motion (2.5) and the Maurer-Cartan equation $d J+J \wedge J=0$.

We often discuss the $C \rightarrow 0$ limit, which corresponds to the $\alpha \rightarrow 0$ limit from (2.7). Before taking the limit, we have to rescale $\lambda_{R}$ as

$$
\lambda_{R}=\alpha \tilde{\lambda}_{R}
$$

Then the $\alpha \rightarrow 0$ limit of (2.6) leads to the Lax pair of rational type for $S U(2)_{\mathrm{R}}$.

It is convenient later to use the light-cone notation like

$$
\begin{aligned}
L_{ \pm}^{R}\left(x ; \lambda_{R}\right) & =L_{t}^{R}\left(x ; \lambda_{R}\right) \pm L_{x}^{R}\left(x ; \lambda_{R}\right) \\
& =-\frac{\sinh \alpha}{\sinh \left(\alpha \pm \lambda_{R}\right)}\left[T^{-} J_{ \pm}^{+}+T^{+} J_{ \pm}^{-}+\frac{\cosh \left(\alpha \pm \lambda_{R}\right)}{\cosh \alpha} T^{3} J_{ \pm}^{3}\right]
\end{aligned}
$$

where $T^{1,2}$ are recombined into

$$
T^{ \pm} \equiv \frac{1}{\sqrt{2}}\left(T^{1} \pm i T^{2}\right)=T_{\mp}
$$

Since the Lax pair given in (2.6) has the periodicity $2 \pi i$ with $\lambda_{R}$ by the definition

$$
L_{ \pm}^{R}\left(x ; \lambda_{R}\right)=L_{ \pm}^{R}\left(x ; \lambda_{R}+2 \pi i\right)
$$

the spectral parameter $\lambda_{R}$ can be regarded as living on a cylinder. For our convention, the cylinder is parametrized by

$$
-\frac{\pi}{2}<\operatorname{Im} \lambda_{R} \leq \frac{3}{2} \pi
$$

The Lax pair (2.6) allows $\left|\lambda_{R}\right|=\infty$ but has four poles ${ }^{5}$,

$$
\lambda_{R}= \pm \alpha, \quad \pm \alpha+\pi i
$$




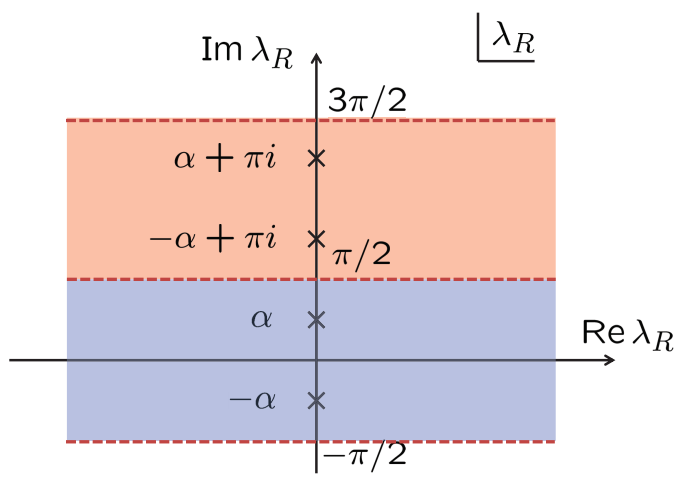

a) For $C>0$

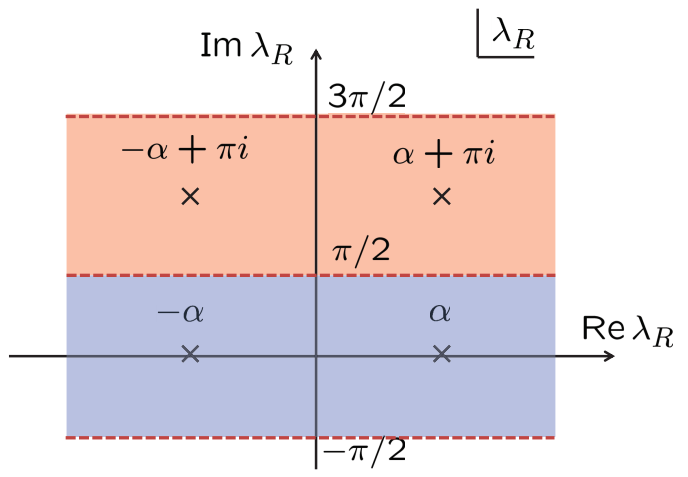

b) For $-1<C<0$

Figure 1. $\lambda_{R}$ takes values on a cylinder with four punctures.

Thus the cylinder has four punctures as depicted in Figure 1.

It is useful to introduce a new parameter defined as

$$
z_{R} \equiv \mathrm{e}^{-\lambda_{R}} .
$$

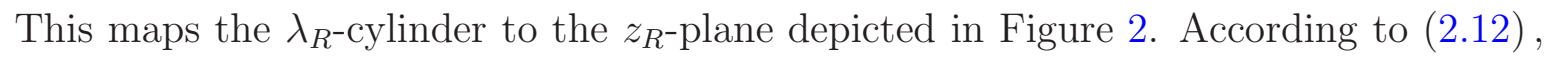
the argument of $z_{R}$ satisfies

$$
-\frac{3}{2} \pi \leq \arg \left(z_{R}\right)<\frac{\pi}{2}
$$

With the spatial component of the Lax pair (2.10), the associated monodromy matrix is constructed as

$$
U^{R}\left(\lambda_{R}\right)=\mathrm{P} \exp \left[\int_{-\infty}^{\infty} d x L_{x}^{R}\left(x ; \lambda_{R}\right)\right],
$$

where the symbol $\mathrm{P}$ is the path-ordering. Because of the flatness condition (2.8), this quantity is conserved

$$
\frac{d}{d t} U^{R}\left(\lambda_{R}\right)=0
$$

By expanding $U^{R}\left(\lambda_{R}\right)$ around $\left|z_{R}\right|<1$ and $\left|z_{R}\right|>1$, the generators of quantum affine algebra are obtained at the classical level [17].

In the current algebra, non-ultra local terms are contained as in the case of principal chiral models [22], hence there is a subtlety in computing classical $r$-matrix. We follow the $r / s$-matrix formalism [23] and show the classical integrability ${ }^{6}$.

\footnotetext{
${ }^{5}$ The number of poles is twice in the relativistic theory in comparison to the non-relativistic case.

${ }^{6}$ The monodromy matrices in [16] are computed as the retarded monodromy matrices by following [24]. However, it causes a discrepancy and we have to follow the $r / s$-matrix formalism [23] as in [18].
} 
With the tensor product notation

$$
\{A \stackrel{\otimes}{,} B\}_{\mathrm{P}} \equiv\{A \otimes 1,1 \otimes B\}_{\mathrm{P}},
$$

the Poisson bracket of the spatial components of the Lax pair is given by

$$
\begin{aligned}
\left\{L_{x}^{R}\left(x ; \lambda_{R}\right) \stackrel{\otimes}{,} L_{x}^{R}\left(y ; \mu_{R}\right)\right\}_{\mathrm{P}}= & {\left[r^{R}\left(\lambda_{R}, \mu_{R}\right), L_{x}^{R}\left(x ; \lambda_{R}\right) \otimes 1+1 \otimes L_{x}^{R}\left(y ; \mu_{R}\right)\right] \delta(x-y) } \\
& -\left[s^{R}\left(\lambda_{R}, \mu_{R}\right), L_{x}^{R}\left(x ; \lambda_{R}\right) \otimes 1-1 \otimes L_{x}^{R}\left(y ; \mu_{R}\right)\right] \delta(x-y) \\
& -2 s^{R}\left(\lambda_{R}, \mu_{R}\right) \partial_{x} \delta(x-y) .
\end{aligned}
$$

The classical $r$-matrix $r^{L}\left(\lambda_{R}, \mu_{R}\right)$ and $s$-matrix $s^{L}\left(\lambda_{R}, \mu_{R}\right)$ are given by [18]

$$
\begin{aligned}
& r^{R}\left(\lambda_{R}, \mu_{R}\right) \equiv \frac{h^{R}\left(\lambda_{R}\right)+h^{R}\left(\mu_{R}\right)}{2 \sinh \left(\lambda_{R}-\mu_{R}\right)}\left(T^{+} \otimes T^{-}+T^{-} \otimes T^{+}+\cosh \left(\lambda_{R}-\mu_{R}\right) T^{3} \otimes T^{3}\right), \\
& s^{R}\left(\lambda_{R}, \mu_{R}\right) \equiv \frac{h^{R}\left(\lambda_{R}\right)-h^{R}\left(\mu_{R}\right)}{2 \sinh \left(\lambda_{R}-\mu_{R}\right)}\left(T^{+} \otimes T^{-}+T^{-} \otimes T^{+}+\cosh \left(\lambda_{R}-\mu_{R}\right) T^{3} \otimes T^{3}\right),
\end{aligned}
$$

where a new function $h^{R}\left(\lambda_{R}\right)$ is defined as

$$
h^{R}\left(\lambda_{R}\right) \equiv \frac{\sinh \alpha \cosh \alpha \sinh ^{2} \lambda_{R}}{\sinh \left(\alpha-\lambda_{R}\right) \sinh \left(\alpha+\lambda_{R}\right)} .
$$

It is easy to show the extended classical Yang-Baxter equation is satisfied,

$$
\left[(r+s)_{13}^{R}(\lambda, \nu),(r-s)_{12}^{R}(\lambda, \mu)\right]+\left[(r+s)_{23}^{R}(\mu, \nu),(r+s)_{12}^{R}(\lambda, \mu)\right]
$$

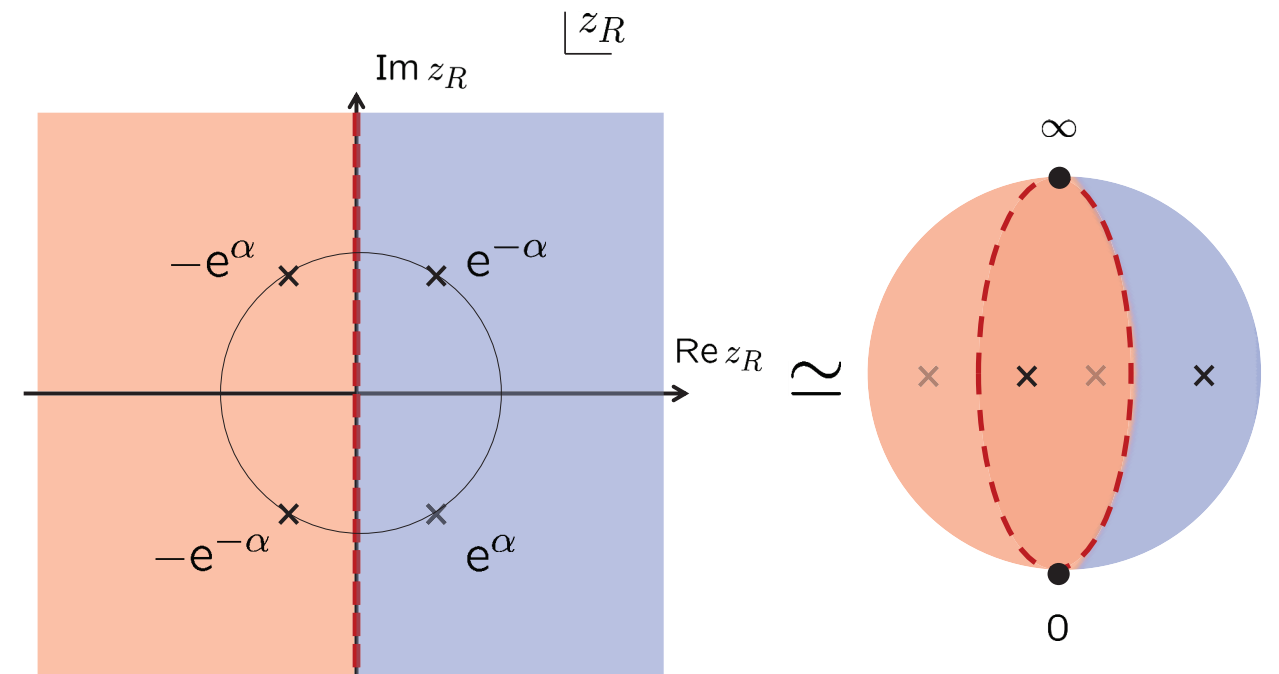

Figure 2. The $z_{R}$-plane for $C>0$ is depicted. It has four punctures but contains $\infty$. Hence this plane should be regarded as a Riemann sphere with four punctures. 


$$
+\left[(r+s)_{23}^{R}(\mu, \nu),(r+s)_{13}^{R}(\lambda, \nu)\right]=0,
$$

where the subscripts denote the vector spaces on which the $r / s$-matrices act.

Finally we comment on the pole structure of the $r / s$-matrices. There are two kinds of poles there. The first is the four poles of $h^{R}\left(\lambda_{R}\right)$ given in (2.15) that exactly agrees with those of the Lax pair in (2.6). The second is the two poles coming from the factor $1 / \sinh \left(\lambda_{R}-\mu_{R}\right)$ in the $r$-matrix, $\lambda_{R}=\mu_{R}$ and $\lambda_{R}=\mu_{R}+\pi i\left(\right.$ or $\left.\lambda_{R}=\mu_{R}-\pi i\right)$, depending on the location of $\mu_{R}$. Note that there is no pole of the second kind in the $s$-matrix. In order for the $r / s$-matrices to satisfy the Yang-Baxter equation (2.16), the detail form in (2.15) is irrelevant. Therefore we distinguish the class of $r$-matrix in terms of the number of poles in the $r$-matrix apart from the pole coming from the scalar function (2.15). This classification is the same as the one in [25]. According to this criterion, the $r$-matrix in the present case is of trigonometric type.

\subsection{Rational description}

The other is the rational description, which has been developed in [14], based on the Yangian algebra.

Constructing the Lax pairs in this description, we use the improved currents,

$$
j_{\mu}^{L_{ \pm}}=g J_{\mu} g^{-1}-2 C \operatorname{Tr}\left(T^{3} J_{\mu}\right) g T^{3} g^{-1} \mp \sqrt{C} \epsilon_{\mu \nu} \partial^{\nu}\left(g T^{3} g^{-1}\right) .
$$

The anti-symmetric tensor $\epsilon_{\mu \nu}$ is normalized with $\epsilon_{t x}=1$. The third term is the improvement term so that the currents (2.17) satisfy the flatness condition [14]

$$
\epsilon^{\mu \nu}\left(\partial_{\mu} j_{\nu}^{L_{ \pm}}-j_{\mu}^{L_{ \pm}} j_{\nu}^{L_{ \pm}}\right)=0
$$

There are two types of currents depending on the sign of the improvement term and the subscript of $L_{ \pm}$in $(2.17)$ denotes it.

It is worth noting that, with the improved currents (2.17), the classical action (2.4) can be rewritten into a simple, dipole-like form,

$$
S=\frac{1}{1+C} \int_{-\infty}^{\infty} d t \int_{-\infty}^{\infty} d x \eta^{\mu \nu} \operatorname{Tr}\left(j_{\mu}^{L_{+}} j_{\nu}^{L_{-}}\right) .
$$

We have not realized the advantage of this expression so far, but it looks very suggestive.

With the improved currents (2.17), two kinds of Lax pairs are constructed.

The one is a Lax pair represented by $j_{\mu}^{L_{+}}$,

$$
L_{t}^{L_{+}}\left(x ; \lambda_{L_{+}}\right) \equiv \frac{1}{1-\lambda_{L_{+}}^{2}}\left(j_{t}^{L_{+}}-\lambda_{L_{+}} j_{x}^{L_{+}}\right)
$$




$$
\begin{aligned}
L_{x}^{L_{+}}\left(x ; \lambda_{L_{+}}\right) & \equiv \frac{1}{1-\lambda_{L_{+}}^{2}}\left(j_{x}^{L_{+}}-\lambda_{L_{+}} j_{t}^{L_{+}}\right) \\
L_{ \pm}^{L_{+}}\left(x ; \lambda_{L_{+}}\right) & \equiv L_{t}^{L_{+}}\left(x ; \lambda_{L_{+}}\right) \pm L_{x}^{L_{+}}\left(x ; \lambda_{L_{+}}\right)=\frac{1}{1 \pm \lambda_{L_{+}}} j_{ \pm}^{L_{+}} \\
& =\frac{1}{1 \pm \lambda_{L_{+}}} g\left(J_{ \pm}-2 C \operatorname{Tr}\left(T^{3} J_{ \pm}\right) T^{3} \mp \sqrt{C}\left[J_{ \pm}, T^{3}\right]\right) g^{-1} .
\end{aligned}
$$

The terms including $\sqrt{C}$ come from the improvement term. The spectral parameter $\lambda_{L_{+}}$takes values on a Riemann sphere except the poles $\lambda_{L_{+}}= \pm 1$, namely a two punctured Riemann sphere. It is noted that the zero curvature condition

$$
\left[\partial_{t}-L_{t}^{L_{+}}\left(x ; \lambda_{L_{+}}\right), \partial_{x}-L_{x}^{L_{+}}\left(x ; \lambda_{L_{+}}\right)\right]=0
$$

also leads the equations of motion (2.5) and the flatness condition (2.18).

The associated monodromy matrix

$$
U^{L_{+}}\left(\lambda_{L_{+}}\right)=\mathrm{P} \exp \left[\int_{-\infty}^{\infty} d x L_{x}^{L_{+}}\left(x ; \lambda_{L_{+}}\right)\right]
$$

is also conserved due to the zero curvature condition (2.21) as

$$
\frac{d}{d t} U^{L_{+}}\left(\lambda_{L_{+}}\right)=0 .
$$

The generators of Yangian are obtained by expanding this monodromy matrix around $\lambda_{L_{+}}=\infty$ [14]. The Poisson bracket of the spatial components of the Lax pair leads to the $r / s$-matrices,

$$
\begin{aligned}
& r^{L}\left(\lambda_{L_{+}}, \mu_{L_{+}}\right)=\frac{h^{L}\left(\lambda_{L_{+}}\right)+h^{L}\left(\mu_{L_{+}}\right)}{2\left(\lambda_{L_{+}}-\mu_{L_{+}}\right)}\left(T^{+} \otimes T^{-}+T^{-} \otimes T^{+}+T^{3} \otimes T^{3}\right), \\
& s^{L}\left(\lambda_{L_{+}}, \mu_{L_{+}}\right)=\frac{h^{L}\left(\lambda_{L_{+}}\right)-h^{L}\left(\mu_{L_{+}}\right)}{2\left(\lambda_{L_{+}}-\mu_{L_{+}}\right)}\left(T^{+} \otimes T^{-}+T^{-} \otimes T^{+}+T^{3} \otimes T^{3}\right),
\end{aligned}
$$

where a scalar function $h^{L}\left(\lambda_{L_{+}}\right)$is defined as

$$
h^{L}\left(\lambda_{L_{+}}\right) \equiv \frac{C+\lambda_{L_{+}}^{2}}{1-\lambda_{L_{+}}^{2}} .
$$

The $r$-matrix function has a single pole apart from the poles of $h^{L}\left(\lambda_{L_{+}}\right)$. Hence the $r / s$-matrices are of rational type in the sense of [25]. They satisfy the extended YangBaxter equation (2.16) . 
The other Lax pair with $j_{\mu}^{L_{-}}$is given by

$$
\begin{aligned}
L_{t}^{L_{-}}\left(x ; \lambda_{L_{-}}\right) & \equiv \frac{1}{1-\lambda_{L_{-}}^{2}}\left(j_{t}^{L_{-}}-\lambda_{L_{-}} j_{x}^{L_{-}}\right) \\
L_{x}^{L_{-}}\left(x ; \lambda_{L_{-}}\right) & \equiv \frac{1}{1-\lambda_{L_{-}}^{2}}\left(j_{x}^{L_{-}}-\lambda_{L_{-}} j_{t}^{L_{-}}\right) \\
L_{ \pm}^{L_{-}}\left(x ; \lambda_{L_{-}}\right) & \equiv L_{t}^{L_{-}}\left(x ; \lambda_{L_{-}}\right) \pm L_{x}^{L_{-}}\left(x ; \lambda_{L_{-}}\right)=\frac{1}{1 \pm \lambda_{L_{-}}} j_{ \pm}^{L_{-}} \\
& =\frac{1}{1 \pm \lambda_{L_{-}}} g\left(J_{ \pm}-2 C \operatorname{Tr}\left(T^{3} J_{ \pm}\right) T^{3} \pm \sqrt{C}\left[J_{ \pm}, T^{3}\right]\right) g^{-1}
\end{aligned}
$$

The spectral parameter $\lambda_{L_{-}}$is independent of $\lambda_{L_{+}}$and $\lambda_{L_{-}}$takes values on another Riemann sphere with two punctures. The zero curvature condition is given by

$$
\left[\partial_{t}-L_{t}^{L_{-}}\left(x ; \lambda_{L_{-}}\right), \partial_{x}-L_{x}^{L_{-}}\left(x ; \lambda_{L_{-}}\right)\right]=0 .
$$

This Lax pair also leads to the equations of motion (2.5) and the flatness condition $(2.18)$.

The monodromy matrix is constructed as

$$
U^{L_{-}}\left(\lambda_{L_{-}}\right)=\mathrm{P} \exp \left[\int_{-\infty}^{\infty} d x L_{x}^{L_{-}}\left(x ; \lambda_{L_{-}}\right)\right] .
$$

Similarly, this is also a conserved quantity because of the zero curvature condition (2.25) and the Poisson bracket of the spatial components of the Lax pair leads to the $r / s$-matrices of rational class. The resulting $r / s$-matrices are the same as those of $L_{x}^{L_{+}}\left(x ; \lambda_{L_{+}}\right)$, up to the spectral parameters. It is a convincing result because the $r / s$-matrices depend only on $C$, not on $\sqrt{C}$.

In the following, we will argue that the monodromy matrices introduced in (2.14), (2.22) and (2.26) are gauge-equivalent under a certain relation of spectral parameters and the rescalings of $\operatorname{sl}(2)$ generators.

\section{The trigonometric/rational correspondence}

In order to discuss the direct relations among monodromy matrices, we would like to see the correspondence between the trigonometric and rational descriptions by expanding the monodromy matrices around some points. The data on the expansion points is enough to determine the relation of spectral parameters. The necessity of the rescaling of $\operatorname{sl}(2)$ generators is anticipated in comparison to the level structure of quantum affine algebra. 


\subsection{Expansions of $U^{R}\left(\lambda_{R}\right)$}

Let us consider expanding the monodromy matrix $U^{R}\left(\lambda_{R}\right)$ in (2.14) around some points.

The first is the expansion around $\left|z_{R}\right|<1$ and $\left|z_{R}\right|>1$. In these regimes, $U^{R}\left(\lambda_{R}\right)$ is expanded like [17]

$$
\begin{array}{ll}
U^{R}\left(\lambda_{R}\right)=\mathrm{e}^{\bar{u}_{0}} \exp \left[\sum_{n=1}^{\infty} z_{R}^{n} \bar{u}_{n}\right] & \left(\left|z_{R}\right|<1\right), \\
U^{R}\left(\lambda_{R}\right)=\mathrm{e}^{u_{0}} \exp \left[\sum_{n=1}^{\infty} z_{R}^{-n} u_{n}\right] & \left(\left|z_{R}\right|>1\right) .
\end{array}
$$

Here $u_{n}$ and $\bar{u}_{m}(n, m=0,1, \ldots)$ consist of conserved charges. For example, the first two of them are

$$
\begin{aligned}
& u_{0}=-\bar{u}_{0}=i \gamma T^{3} Q_{(0)}^{R, 3}, \quad \gamma \equiv \frac{\sqrt{C}}{1+C}, \\
& u_{1}=2 i \gamma\left(T^{-} \mathrm{e}^{\gamma Q_{(0)}^{R, 3} / 2} Q_{(1)}^{R,+}+T^{+} \mathrm{e}^{-\gamma Q_{(0)}^{R, 3} / 2} \widetilde{Q}_{(1)}^{R,-}\right) \\
& \bar{u}_{1}=-2 i \gamma\left(T^{+} \mathrm{e}^{\gamma Q_{(0)}^{R, 3} / 2} Q_{(1)}^{R,-}+T^{-} \mathrm{e}^{-\gamma Q_{(0)}^{R, 3} / 2} \widetilde{Q}_{(1)}^{R,+}\right) .
\end{aligned}
$$

The conserved charges $Q_{(0)}^{R, 3}, Q_{(1)}^{R, \pm}$ and $\widetilde{Q}_{(1)}^{R, \pm}$ precisely generate a quantum affine algebra $U_{q}\left(\widehat{\operatorname{sl}(2)_{\mathrm{R}}}\right)$ in the sense of Drinfeld's first realization [26]. The expressions of the charges are given in [17]. ${ }^{7}$ Note that $\gamma$ is related to a $q$-deformation parameter in $U_{q}\left(s l(2)_{\mathrm{R}}\right)$ $[26,27]$ through the relation $q \equiv \mathrm{e}^{\gamma}[16]$.

The second is the expansion around $\lambda_{R}=0$. The spatial component of the Lax pair is expanded around $\lambda_{R}=0$ as

$$
\begin{aligned}
L_{x}^{R}\left(x ; \lambda_{R}\right)= & -J_{x}+\lambda_{R}\left(-\frac{i}{\sqrt{C}} J_{t}+2 i \sqrt{C} \operatorname{Tr}\left(T^{3} J_{t}\right) T^{3}\right) \\
& +\lambda_{R}^{2}\left[\left(\frac{1}{C}+\frac{1}{2}\right) J_{x}-\frac{1}{2} \operatorname{Tr}\left(T^{3} J_{x}\right) T^{3}\right]+\mathcal{O}\left(\lambda_{R}^{3}\right) .
\end{aligned}
$$

This leads to the expansion of $U^{R}\left(\lambda_{R}\right)$ around $\lambda_{R}=0$,

$$
U^{R}\left(\lambda_{R}\right)=g_{\infty}^{-1} \cdot \exp \left[\sum_{n=0}^{\infty}\left(-\frac{i \lambda_{R}}{\sqrt{C}}\right)^{n+1} Q_{(n)}^{L}\right] \cdot g_{\infty} .
$$

Here $Q_{(n)}^{L}(n=0,1, \ldots)$ are the conserved charges because $U^{R}\left(\lambda_{R}\right)$ is a conserved quantity. The first two charges $Q_{(0)}^{L}$ and $Q_{(1)}^{L}$ generate the $S U(2)_{\mathrm{L}}$ Yangian in the sense of Drinfeld's first realization [26].

\footnotetext{
$7 \mathrm{abc}$
} 
Before mentioning $Q_{(0)}^{L}$ and $Q_{(1)}^{L}$, we have to detail the construction of the Yangian generators. By using the improved $S U(2)_{\mathrm{L}}$ currents $(2.17)$, two kinds of the generators can be constructed as

$$
\begin{aligned}
Q_{(0)}^{L_{ \pm}} & =\int_{-\infty}^{\infty} d x j_{t}^{L_{ \pm}}(x), \\
Q_{(1)}^{L_{ \pm}} & =\frac{1}{2} \int_{-\infty}^{\infty} d x \int_{-\infty}^{\infty} d y \epsilon(x-y) j_{t}^{L_{ \pm}}(x) j_{t}^{L_{ \pm}}(y)-\int_{-\infty}^{\infty} d x j_{x}^{L_{ \pm}}(x),
\end{aligned}
$$

where the signature function $\epsilon(x-y) \equiv \theta(x-y)-\theta(y-x)$ and $\theta(x)$ is a step function. Note that the concrete expressions do not depend on $\sqrt{C}$ and hence it is shown that

$$
Q_{(0)}^{L_{+}}=Q_{(0)}^{L_{-}} \quad Q_{(1)}^{L_{+}}=Q_{(1)}^{L_{-}} .
$$

This is the case for higher conserved charges. Thus either of $Q_{(n)}^{L_{+}}$and $Q_{(n)}^{L_{-}}$may be taken as $Q_{(n)}^{L}$ in (3.3). For later discussion, we choose $Q_{(n)}^{L_{+}}$as $Q_{(n)}^{L}$ here.

It is quite non-trivial that the $S U(2)_{L}$ Yangian generators have been reproduced by expanding $U^{R}\left(\lambda_{R}\right)$ around $\lambda_{R}=0$, because $U^{R}\left(\lambda_{R}\right)$ leads to the trigonometric $r / s$-matrices while the Yangian is closely related to the rational class. Conversely, we show that a quantum affine algebra is reproduced by expanding $U^{L_{ \pm}}\left(\lambda_{L_{ \pm}}\right)$in the next subsection.

Finally, let us consider the expansion around $\lambda_{R}=\pi i$. It also provides the $S U(2)_{\mathrm{L}}$ Yangian generators, basically because the Lax pair is invariant under the shift of $\lambda_{R}$ by $\pi i$, up to the sign flipping of $T^{ \pm}$,

$$
L_{ \pm}^{R}\left(x ; \lambda_{R}+\pi i\right)=-\frac{\sinh \alpha}{\sinh \left(\alpha \pm \lambda_{R}\right)}\left[-T^{-} J_{ \pm}^{+}-T^{+} J_{ \pm}^{-}+\frac{\cosh \left(\alpha \pm \lambda_{R}\right)}{\cosh \alpha} T^{3} J_{ \pm}^{3}\right] .
$$

This sign flipping is closely related to the rescalings of $s l(2)$ generators discussed later. The Yangian charges in this expansion should be identified with $Q_{(n)}^{L_{-}}$, according to the choice in the expansion around $\lambda_{R}=0$. The reason to assign the charges in this way will be clarified later.

\subsection{Expansions of $U^{L_{ \pm}}\left(\lambda_{L_{ \pm}}\right)$}

We then consider the expansions of $U^{L_{+}}\left(\lambda_{L_{+}}\right)$and $U^{L_{-}}\left(\lambda_{L_{-}}\right)$around some points.

\section{Expanding $U^{L_{+}}\left(\lambda_{L_{+}}\right)$}

The first is the expansion of $U^{L_{+}}\left(\lambda_{L_{+}}\right)$around $\lambda_{L_{+}}=\infty$, where the $S U(2)_{\mathrm{L}}$ Yangian generators are obtained as $[14,16]$

$$
U^{L_{+}}\left(\lambda_{L_{+}}\right)=\exp \left[\sum_{n=0}^{\infty} \lambda_{L_{+}}^{-n-1} Q_{(n)}^{L}\right] .
$$


The charges $Q_{(n)}^{L}$ here are $Q_{(n)}^{L_{+}}$by definition, and the expansion in (3.7) exactly agrees with the expansion of $U^{R}\left(\lambda_{R}\right)$ around $\lambda_{R}=0$.

Next let us consider the expansion around $\lambda_{L_{+}}= \pm i \sqrt{C}$. It is convenient to introduce infinitesimal parameters $\epsilon_{( \pm)}^{+}$as

$$
\epsilon_{( \pm)}^{+} \equiv \lambda_{L_{+}} \mp i \sqrt{C} \text {. }
$$

The expansions of $L_{x}^{L_{+}}\left(x ; \lambda_{L_{+}}\right)$with respect to $\epsilon_{( \pm)}^{+}$are given by, respectively,

$$
\begin{aligned}
L_{x}^{L_{+}}\left(x ; \lambda_{L_{+}}\right)= & g J_{x} g^{-1}+g\left[\mp i \sqrt{C} T^{3} J_{t}^{3} \mp \frac{2 i \sqrt{C}}{1+C} T^{ \pm}\left(J_{t}^{\mp} \mp i \sqrt{C} J_{x}^{\mp}\right)\right] g^{-1} \\
& -\frac{\epsilon_{( \pm)}^{+}}{1+C} g\left[T^{ \pm}\left(\frac{1-C}{1+C}\left(J_{t}^{\mp} \mp i \sqrt{C} J_{x}^{\mp}\right) \mp \frac{2 i \sqrt{C}}{1+C}\left(J_{x}^{\mp} \mp i \sqrt{C} J_{t}^{\mp}\right)\right)\right. \\
& \left.+T^{\mp}\left(J_{t}^{ \pm} \mp i \sqrt{C} J_{x}^{ \pm}\right)+T^{3}\left((1-C) J_{t}^{3} \mp 2 i \sqrt{C} J_{x}^{3}\right)\right] g^{-1}+\mathcal{O}\left(\left(\epsilon_{( \pm)}^{+}\right)^{2}\right) .
\end{aligned}
$$

It would be helpful to introduce the following identity

$$
\begin{aligned}
& \mathrm{P} \exp \left[\int_{\beta}^{\alpha} d x\left(T^{3} \partial_{x} \phi^{3}+T^{+} L_{x}^{-}+T^{-} L_{x}^{+}\right)(x)\right] \\
= & \mathrm{e}^{T^{3} \phi^{3}(\alpha)} \mathrm{P} \exp \left[\int_{\beta}^{\alpha} d x\left(T^{+} \mathrm{e}^{+i \phi^{3}} L_{x}^{-}+T^{-} \mathrm{e}^{-i \phi^{3}} L_{x}^{+}\right)(x)\right] \mathrm{e}^{-T^{3} \phi^{3}(\beta)} .
\end{aligned}
$$

This identity is used in the following step.

The expansion (3.8) and the identity (3.9) lead to the monodromy matrix $U^{L_{+}}\left(\lambda_{L_{+}}\right)$ in terms of $\epsilon_{(+)}^{+}$,

$$
U^{L_{+}}\left(\lambda_{L_{+}}\right)=g_{\infty} \cdot \bar{v}_{(0)}^{+} \exp \left[\sum_{n=1}^{\infty}\left(-\frac{\epsilon_{(+)}^{+}}{1+C}\right)^{n} \bar{v}_{(n)}^{+}\right] \cdot g_{\infty}^{-1},
$$

where $\bar{v}_{(n)}^{+}(n=0,1, \ldots)$ consist of the conserved charges. The first two of them are represented by $Q_{(0)}^{R, 3}, Q_{(1)}^{R,-}$ and $\widetilde{Q}_{(1)}^{R,+}$ as follows:

$$
\begin{aligned}
& \bar{v}_{(0)}^{+}=\mathrm{e}^{-i \gamma T^{3} Q_{(0)}^{R, 3}} \exp \left[-2 i \gamma T^{+} \mathrm{e}^{\gamma Q_{(0)}^{R, 3} / 2} Q_{(1)}^{R,-}\right], \\
& \bar{v}_{(1)}^{+}=T^{-} \mathrm{e}^{-\gamma Q_{(0)}^{R, 3} / 2} \widetilde{Q}_{(1)}^{R,+}-\gamma T^{3}\left[\bar{Q}_{(2)}^{R, 3}-Q_{(1)}^{R,-} \widetilde{Q}_{(1)}^{R,+}\right] \\
& -\gamma^{2} T^{+} \mathrm{e}^{\gamma Q_{(0)}^{R, 3} / 2}\left[Q_{(3)}^{R,-}-\bar{Q}_{(2)}^{R, 3} Q_{(1)}^{R,-}+\frac{2}{3}\left(Q_{(1)}^{R,-}\right)^{2} \widetilde{Q}_{(1)}^{R,+}\right] .
\end{aligned}
$$


Similarly, the expansion in terms of $\epsilon_{(-)}^{-}$is given by

$$
U^{L_{+}}\left(\lambda_{L_{+}}\right)=g_{\infty} \cdot v_{(0)}^{+} \exp \left[\sum_{n=1}^{\infty}\left(-\frac{\epsilon_{(-)}^{+}}{1+C}\right)^{n} v_{(n)}^{+}\right] \cdot g_{\infty}^{-1},
$$

where $v_{(n)}^{+}(n=0,1, \ldots)$ also consist of the conserved charges and the first two are expressed with $Q_{(0)}^{R, 3}, Q_{(1)}^{R,+}$ and $\widetilde{Q}_{(1)}^{R,-}$ like

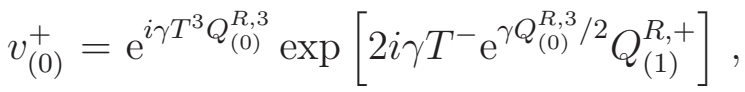

$$
\begin{aligned}
& v_{(1)}^{+}=T^{+} \mathrm{e}^{-\gamma Q_{(0)}^{R, 3} / 2} \widetilde{Q}_{(1)}^{R,-}-\gamma T^{3}\left[Q_{(2)}^{R, 3}-Q_{(1)}^{R,+} \widetilde{Q}_{(1)}^{R,-}\right] \\
& -\gamma^{2} T^{-} \mathrm{e}^{\gamma Q_{(0)}^{R, 3} / 2}\left[Q_{(3)}^{R,+}-Q_{(2)}^{R, 3} Q_{(1)}^{R,+}+\frac{2}{3}\left(Q_{(1)}^{R,+}\right)^{2} \widetilde{Q}_{(1)}^{R,-}\right] .
\end{aligned}
$$

In summary, all of the generators of quantum affine algebra have been obtained by expanding $U^{L_{+}}\left(\lambda_{L_{+}}\right)$around $\lambda_{L_{+}}= \pm i \sqrt{C}$. This result is also far from trivial because $U^{L_{+}}\left(\lambda_{L_{+}}\right)$yields the rational $r / s$-matrices while quantum affine algebra is associated with the trigonometric class.

Expanding $U^{L_{-}}\left(\lambda_{L_{-}}\right)$

It is a turn to discuss the expansions of $U^{L_{-}}\left(\lambda_{L_{-}}\right)$. We first consider he expansion around $\lambda_{L_{-}}=\infty$, where the $S U(2)_{\mathrm{L}}$ Yangian generators are obtained as $[14,16]$

$$
U^{L_{-}}\left(\lambda_{L_{-}}\right)=\exp \left[\sum_{n=0}^{\infty}\left(\lambda_{L_{-}}\right)^{-n-1} Q_{(n)}^{L}\right] .
$$

The charges obtained here are $Q_{(n)}^{L_{-}}$by definition, and the expansion in (3.14) exactly agrees with the expansion of $U^{R}\left(\lambda_{R}\right)$ around $\lambda_{R}=\pi i$.

Then let us consider the expansion around $\lambda_{L_{-}}= \pm i \sqrt{C}$. It is convenient to introduce infinitesimal parameters

$$
\epsilon_{( \pm)}^{-} \equiv \lambda_{L_{-}} \mp i \sqrt{C}
$$

The charges $Q_{(0)}^{R, 3}, Q_{(1)}^{R,-}$ and $\widetilde{Q}_{(1)}^{R,+}$ are obtained from the expansion in terms of $\epsilon_{(+)}^{-}$like

$$
U^{L_{-}}\left(\lambda_{L_{-}}\right)=g_{\infty} \cdot v_{(0)}^{-} \exp \left[\sum_{n=1}^{\infty}\left(-\frac{\epsilon_{(+)}^{-}}{1+C}\right)^{n} v_{(n)}^{-}\right] \cdot g_{\infty}^{-1}
$$

where $v_{(n)}^{-}(n=0,1, \ldots)$ consist of the conserved charges. The first two are given by

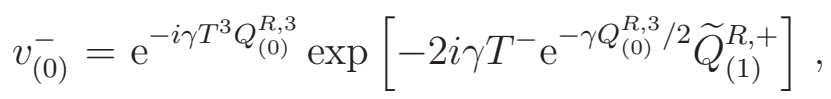




$$
\begin{aligned}
v_{(1)}^{-}= & T^{+} \mathrm{e}^{\gamma Q_{(0)}^{R, 3} / 2} Q_{(1)}^{R,-}-\gamma T^{3}\left[\bar{Q}_{(2)}^{R, 3}+\widetilde{Q}_{(1)}^{R,+} Q_{(1)}^{R,-}\right] \\
& -\gamma^{2} T^{-} \mathrm{e}^{-\gamma Q_{(0)}^{R, 3} / 2}\left[\widetilde{Q}_{(3)}^{R,+}+\bar{Q}_{(2)}^{R, 3} \widetilde{Q}_{(1)}^{R,+}+\frac{2}{3}\left(\widetilde{Q}_{(1)}^{R,+}\right)^{2} Q_{(1)}^{R,-}\right] .
\end{aligned}
$$

The remaining charges $Q_{(0)}^{R, 3}, Q_{(1)}^{R,+}$ and $\widetilde{Q}_{(1)}^{R,-}$ come from the expansion in terms of $\epsilon_{(-)}^{-}$ as

$$
U^{L_{-}}\left(\lambda_{L_{-}}\right)=g_{\infty} \cdot \bar{v}_{(0)}^{-} \exp \left[\sum_{n=1}^{\infty}\left(-\frac{\epsilon_{(-)}^{-}}{1+C}\right)^{n} \bar{v}_{(n)}^{-}\right] \cdot g_{\infty}^{-1},
$$

where $\bar{v}_{(n)}^{-}(n=0,1, \ldots)$ consist of the conserved charges. The first two are

$$
\begin{aligned}
& \bar{v}_{(0)}^{-}=\mathrm{e}^{i \gamma T^{3} Q_{(0)}^{R, 3}} \exp \left[2 i \gamma T^{+} \mathrm{e}^{-\gamma Q_{(0)}^{R, 3} / 2} \widetilde{Q}_{(1)}^{R,-}\right], \\
& \bar{v}_{(1)}^{-}=T^{-} \mathrm{e}^{\gamma Q_{(0)}^{R, 3} / 2} Q_{(1)}^{R,+}-\gamma T^{3}\left[Q_{(2)}^{R, 3}+\widetilde{Q}_{(1)}^{R,-} Q_{(1)}^{R,+}\right] \\
& -\gamma^{2} T^{+} \mathrm{e}^{-\gamma Q_{(0)}^{R, 3} / 2}\left[\widetilde{Q}_{(3)}^{R,-}+Q_{(2)}^{R, 3} \widetilde{Q}_{(1)}^{R,-}+\frac{2}{3}\left(\widetilde{Q}_{(1)}^{R,-}\right)^{2} Q_{(1)}^{R,+}\right] .
\end{aligned}
$$

The above results can also be obtained by flipping the sign of $\sqrt{C}$ in the results on $U^{L_{+}}\left(\lambda_{L_{+}}\right)$. Under the sign flipping, $Q_{(0)}^{R, 3}$ is invariant while $Q_{(1)}^{R, \pm}$ and $\widetilde{Q}_{(1)}^{R, \pm}$ are mapped each other.

Finally the results obtained here are summarized in Table 1.

\begin{tabular}{c||c|c|c}
\hline Charges $\backslash$ Monodromies & $U^{R}\left(\lambda_{R}\right)$ & $U^{L_{+}}\left(\lambda_{L_{+}}\right)$ & $U^{L_{-}}\left(\lambda_{L_{-}}\right)$ \\
\hline \hline$Q_{(0)}^{R, 3}, Q_{(1)}^{R,-}, \widetilde{Q}_{(1)}^{R,+}$ & 0 & $+i \sqrt{C}$ & $+i \sqrt{C}$ \\
\hline$Q_{(0)}^{R, 3}, Q_{(1)}^{R,+}, \widetilde{Q}_{(1)}^{R,-}$ & $\infty$ & $-i \sqrt{C}$ & $-i \sqrt{C}$ \\
\hline$Q_{(0)}^{L, a}, Q_{(1)}^{L, a}$ & \pm 1 & $\infty$ & $\infty$ \\
\hline \hline local charges & $\pm \mathrm{e}^{\alpha}, \pm \mathrm{e}^{-\alpha}$ & \pm 1 & \pm 1 \\
\hline \hline
\end{tabular}

Table 1. The conserved charges and the expansion points of monodromy matrices are listed. For quantum affine algebra and Yangian, the charges are denoted in the sense of Drinfeld's first realization. The expansion points of $U^{R}\left(\lambda_{R}\right)$ are described in terms of $z_{R}$.

\subsection{The relation of spectral parameters}

Now it is a turn to argue the relation of spectral parameters. We have already prepared the data enough to completely fix it. 
We assume the relation of spectral parameters is given by a Möbius transformation. Taking account of the correspondence in Table 1, the Möbius transformation, which relates the expansion points giving the same conserved charges in each description, is uniquely determined as follows,

$$
z_{R}^{2}=\frac{\lambda_{L}-i \sqrt{C}}{\lambda_{L}+i \sqrt{C}} \quad\left(z_{R} \equiv \mathrm{e}^{-\lambda^{R}}\right),
$$

where $\lambda_{L}\left(=\lambda_{L_{+}}\right.$or $\left.\lambda_{L_{-}}\right)$. As we will show in section 4 , it is noted that the map (3.18) is valid not only on some particular expansion points but also on the whole region of the spectral parameters. In checking the correspondence of local charges, it is helpful to use the formula,

$$
\tanh ^{-1} x=\frac{1}{2} \log \frac{1+x}{1-x}
$$

We should be careful for the parameter range of $z_{R}$. The relation (3.18) contains the square of $z_{R}$ and hence two Riemann spheres of $\lambda_{L}$ are basically necessary so that $z_{R}$ is represented by a single-valued function of $\lambda_{L}$. Each regime of $\lambda_{L_{+}}$and $\lambda_{L_{-}}$has already been fixed on a single Riemann sphere with two punctures from consistency of the Lax pair in the rational description, hence it is not possible to use only either of them. Thus it is necessary to use both $\lambda_{L_{+}}$and $\lambda_{L_{-}}$. After all, $z_{R}$ is expressed as

$$
z_{R}=\left\{\begin{array}{cc}
\left(\frac{\lambda_{L_{+}}-i \sqrt{C}}{\lambda_{L_{+}}+i \sqrt{C}}\right)^{1 / 2} & \left(\operatorname{Re} z_{R}>0\right) \\
-\left(\frac{\lambda_{L_{-}}-i \sqrt{C}}{\lambda_{L_{-}}+i \sqrt{C}}\right)^{1 / 2} & \left(\operatorname{Re} z_{R}<0\right)
\end{array} .\right.
$$

This assignment of $\lambda_{L_{+}}$and $\lambda_{L_{-}}$is compatible with that of $S U(2)_{\mathrm{L}}$ Yangian generators.

In the map (3.19), there is a cut between $+i \sqrt{C}$ and $-i \sqrt{C}$ on each of the Riemann spheres with $\lambda_{L_{+}}$and $\lambda_{L_{-}}$, and the two Riemann spheres are joined there as depicted in Fig. 3. Then this cut corresponds to the imaginary axis of $z_{R}$. In order to see this correspondence, let us rewrite the relation (3.18) as

$$
\lambda_{L}=i \sqrt{C} \frac{1+z_{R}^{2}}{1-z_{R}^{2}},
$$

and parametrize the imaginary axis of $z_{R}$ as

$$
z_{R}= \pm i \mathrm{e}^{-\xi} \quad(\xi \in \mathbb{R})
$$




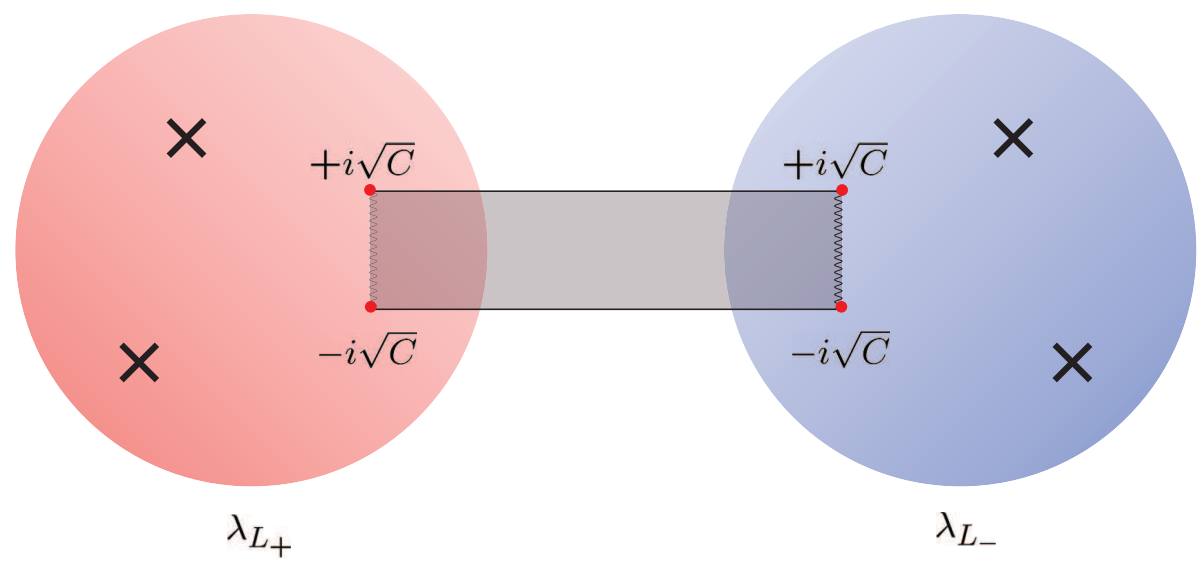

Figure 3. The $\lambda_{L_{ \pm}}$-spheres are joined on the cut between $\pm i \sqrt{C}(C>0)$. The constructed Riemann surface is mapped to the Riemann sphere in $z_{R}$ depicted in Figure 2.

According to the map (3.20), the imaginary axis (3.21) is represented by

$$
\lambda_{L}=i \sqrt{C} \tanh \xi \quad(\xi \in \mathbb{R})
$$

on the $\lambda_{L_{ \pm}}$-spheres. This is nothing but the cut in the map (3.19). More precisely, depending on the sign of $C$, it is written as

$$
\begin{aligned}
& \lambda_{L}=i y, \quad-\sqrt{C}<y<\sqrt{C} \quad(C>0), \\
& \lambda_{L}=x, \quad-\sqrt{|C|}<x<\sqrt{|C|} \quad(-1<C<0) .
\end{aligned}
$$

Thus the resulting Riemann surface described by $\lambda_{L_{+}}$and $\lambda_{L_{-}}$is mapped to the Riemann sphere with $z_{R}$, each other. The number of poles is preserved under the map.

\subsection{The expansions of $U^{L_{ \pm}}\left(\lambda_{L_{ \pm}}\right)$: revisited}

It is worth reconsidering the expansions of monodromy matrices with the relation (3.18).

As a concrete example, we will concentrate on the two expansions,

$$
\begin{array}{cccc}
\text { i) } & U^{L_{+}}\left(\lambda_{L_{+}}\right) & \text {around } & \lambda_{L_{+}}=i \sqrt{C}, \\
\text { ii) } & U^{R}\left(\lambda_{R}\right) & \text { around } & z_{R}=0 .
\end{array}
$$

With the relation (3.18), the expansion parameter $\epsilon_{(+)}^{+}$in the case i) is rewritten as

$$
\epsilon_{(+)}^{+}=\frac{2 i \sqrt{C} z_{R}^{2}}{1-z_{R}^{2}}
$$


Since $\left|\epsilon_{(+)}^{+}\right| \ll 1, z_{R}$ is infinitesimal. Hence $\epsilon_{(+)}^{+}$can be expressed as a power series in $z_{R}$.

With the relation (3.23), the expansion in (3.10) can be rewritten as

$$
\begin{aligned}
& g_{\infty}^{-1} \cdot U^{L_{+}}\left(\lambda_{L_{+}}\right) \cdot g_{\infty} \\
& =\mathrm{e}^{-i \gamma T^{3} Q_{(0)}^{R, 3}} \exp \left[-2 i \gamma T^{+} \mathrm{e}^{\gamma Q_{(0)}^{R, 3} / 2} Q_{(1)}^{R,-}\right] \\
& \times\left\{1-\frac{2 i \sqrt{C} z_{R}^{2}}{1+C}\left[T^{-} \mathrm{e}^{-\gamma Q_{(0)}^{R, 3} / 2} \widetilde{Q}_{(1)}^{R,+}-\gamma T^{3}\left[\bar{Q}_{(2)}^{R, 3}-Q_{(1)}^{R,-} \widetilde{Q}_{(1)}^{R,+}\right]\right.\right. \\
& \left.\left.-\gamma^{2} T^{+} \mathrm{e}^{\gamma Q_{(0)}^{R, 3} / 2}\left[Q_{(3)}^{R,-}-\bar{Q}_{(2)}^{R, 3} Q_{(1)}^{R,-}+\frac{2}{3}\left(Q_{(1)}^{R,-}\right)^{2} \widetilde{Q}_{(1)}^{R,+}\right]\right]+\mathcal{O}\left(z_{R}^{4}\right)\right\} .
\end{aligned}
$$

Notice that the rescaling of $\operatorname{sl}(2)$ generators

$$
T^{ \pm} \rightarrow \mathrm{e}^{\mp \lambda_{R}} T^{ \pm}
$$

makes the expansion in (3.24) into a significant form,

$$
\begin{aligned}
& g_{\infty}^{-1} \cdot U^{L_{+}}\left(\lambda_{L_{+}}\right) \cdot g_{\infty} \\
= & \mathrm{e}^{-i \gamma T^{3} Q_{(0)}^{R, 3}}\left[1-2 i \gamma z_{R} T^{+} \mathrm{e}^{\gamma Q_{(0)}^{R, 3} / 2} Q_{(1)}^{R,-}\right] \\
& \times\left\{1-2 i \gamma z_{R}^{2}\left[z_{R}^{-1} T^{-} \mathrm{e}^{-\gamma Q_{(0)}^{R, 3} / 2} \widetilde{Q}_{(1)}^{R,+}-\gamma T^{3}\left[\bar{Q}_{(2)}^{R, 3}-Q_{(1)}^{R,-} \widetilde{Q}_{(1)}^{R,+}\right]\right.\right. \\
& \left.\left.-\gamma^{2} z_{R} T^{+} \mathrm{e}^{\gamma Q_{(0)}^{R, 3} / 2}\left[Q_{(3)}^{R,-}-\bar{Q}_{(2)}^{R, 3} Q_{(1)}^{R,-}+\frac{2}{3}\left(Q_{(1)}^{R,-}\right)^{2} \widetilde{Q}_{(1)}^{R,+}\right]\right]\right\}+\mathcal{O}\left(z_{R}^{2}\right) \\
= & \mathrm{e}^{-i \gamma T^{3} Q_{(0)}^{R, 3}}\left\{1-2 i \gamma z_{R}\left[T^{+} \mathrm{e}^{\gamma Q_{(0)}^{R, 3} / 2} Q_{(1)}^{R,-}+T^{-} \mathrm{e}^{-\gamma Q_{(0)}^{R, 3} / 2} \widetilde{Q}_{(1)}^{R,+}\right]\right\}+\mathcal{O}\left(z_{R}^{2}\right) .
\end{aligned}
$$

This is nothing but the expansion in the case ii). That is, if the rescaling (3.25) is taken into account, then the expansion in $\epsilon_{(+)}^{+}$can be regarded as the one in $z_{R}$. The rescaling (3.25) is just an isomorphism of the $s l(2)$ algebra, hence it does not mean any modifications of the system.

Similarly, the expansion of $U^{L_{+}}\left(\lambda_{L_{+}}\right)$around $\lambda_{L_{+}}=-i \sqrt{C}$ agrees with that of $U^{R}\left(\lambda_{R}\right)$ around $z_{R}=\infty$ under the relation (3.18) with the rescaling (3.25). In addition, the expansion of $U^{L_{-}}\left(\lambda_{L_{-}}\right)$around $\lambda_{L_{-}}=i \sqrt{C}(-i \sqrt{C})$ agrees with that of $U^{R}\left(\lambda_{R}\right)$ around $z_{R}=0(\infty)$, respectively, if we take another rescaling

$$
T^{ \pm} \rightarrow \mathrm{e}^{ \pm \lambda_{R}} T^{ \pm}
$$

From these agreements, one may anticipate that the rescalings of $s l(2)$ generators

$$
T^{ \pm} \rightarrow \mathrm{e}^{\mp \lambda_{R}} T^{ \pm} \quad \text { for } \quad U^{L_{+}}\left(\lambda_{L_{+}}\right)
$$




$$
T^{ \pm} \rightarrow \mathrm{e}^{ \pm \lambda_{R}} T^{ \pm} \quad \text { for } \quad U^{L_{-}}\left(\lambda_{L_{-}}\right)
$$

would be an important key in arguing the equivalence of monodromy matrices. Indeed this is the case. The rescalings will play an essential role in the next section. Note that the rescalings (3.27) and (3.28) are compatible with the sign flipping in (3.6), because the shift of $\lambda_{R}$,

$$
\lambda_{R} \quad \rightarrow \quad \lambda_{R}+\pi i
$$

flips the sign of $T^{ \pm}$after taking the rescalings (3.27) and (3.28).

\section{Gauge equivalence of monodromy matrices}

Let us consider the gauge-equivalence of monodromy matrices $U^{R}\left(\lambda_{R}\right)$ and $U^{L_{ \pm}}\left(\lambda_{L_{ \pm}}\right)$ under the parameter relation (3.18) and the rescalings (3.27) and (3.28).

\subsection{Gauge equivalence of monodromy matrices: $C=0$}

First of all, as a warming-up, we shall consider the $C=0$ case. This is nothing but the case of $S U(2)$ principal chiral model and its classical integrability is well studied [28-32] (For a comprehensive book, see [33]).

On the one hand, the Lax pair in terms of the right-invariant current

$$
j_{\mu}^{L} \equiv \partial_{\mu} g \cdot g^{-1}=g J_{\mu} g^{-1}
$$

is given by

$$
L_{ \pm}^{L}\left(x ; \lambda_{L}\right)=\frac{1}{1 \pm \lambda_{L}} j_{ \pm}^{L}
$$

where the light-cone components are defined as

$$
L_{t}^{L}\left(x ; \lambda_{L}\right) \equiv \frac{1}{2}\left[L_{+}^{L}\left(x ; \lambda_{L}\right)+L_{-}^{L}\left(x ; \lambda_{L}\right)\right], \quad L_{x}^{L}\left(x ; \lambda_{L}\right) \equiv \frac{1}{2}\left[L_{+}^{L}\left(x ; \lambda_{L}\right)-L_{-}^{L}\left(x ; \lambda_{L}\right)\right] .
$$

On the other hand, the Lax pair in terms of the left-invariant current

$$
j_{\mu}^{R} \equiv-g^{-1} \partial_{\mu} g=-J_{\mu}
$$

is given by

$$
L_{ \pm}^{R}\left(x ; \lambda_{R}\right)=\frac{1}{1 \pm \lambda_{R}} j_{ \pm}^{R}
$$


where the light-components are defined as

$$
L_{t}^{R}\left(x ; \lambda_{R}\right) \equiv \frac{1}{2}\left[L_{+}^{R}\left(x ; \lambda_{R}\right)+L_{-}^{R}\left(x ; \lambda_{R}\right)\right], \quad L_{x}^{R}\left(x ; \lambda_{R}\right) \equiv \frac{1}{2}\left[L_{+}^{R}\left(x ; \lambda_{R}\right)-L_{-}^{R}\left(x ; \lambda_{R}\right)\right] .
$$

Then we may introduce monodromy matrices for the Lax pairs (4.1) and (4.2) like

$$
\begin{aligned}
U^{L}\left(\lambda_{L}\right) & =\mathrm{P} \exp \left[\int_{-\infty}^{\infty} d x L_{x}^{L}\left(x ; \lambda_{L}\right)\right], \\
U^{R}\left(\lambda_{R}\right) & =\mathrm{P} \exp \left[\int_{-\infty}^{\infty} d x L_{x}^{R}\left(x ; \lambda_{R}\right)\right] .
\end{aligned}
$$

From now on, we will show that the two Lax pairs (4.1) and (4.2) are gaugeequivalent under the identification of $\lambda_{L}$ and $\lambda_{R}$ with

$$
\lambda_{L}=\frac{1}{\lambda_{R}}
$$

First of all, let us perform the gauge transformation for the $L_{ \pm}^{L}\left(x ; \lambda_{L}\right)$. Since the Lax pair is transformed as a gauge field, the transformation law is give by

$$
\left[L_{ \pm}^{L}\left(x ; \lambda_{L}\right)\right]^{g} \equiv g^{-1} L_{ \pm}^{L}\left(x ; \lambda_{L}\right) g-g^{-1} \partial_{ \pm} g=-\frac{ \pm \lambda_{L}}{1 \pm \lambda_{L}} J_{ \pm}
$$

Using the relation (4.5), we can show that

$$
\left[L_{ \pm}^{L}\left(x ; \lambda_{L}\right)\right]^{g}=L_{ \pm}^{R}\left(x ; \lambda_{R}\right)
$$

With this relation, we obtain the following formula for covariant derivatives,

$$
g^{-1}\left[\partial_{\mu}-L_{\mu}^{L}\left(x ; \lambda_{L}\right)\right] g=\partial_{\mu}-L_{\mu}^{R}\left(x ; \lambda_{R}\right) .
$$

Thus the transformation law of monodromy matrices is given by

$$
g_{\infty}^{-1} \cdot U^{L}\left(\lambda_{L}\right) \cdot g_{\infty}=U^{R}\left(\lambda_{R}\right)
$$

and we have shown that $U^{L}\left(\lambda_{L}\right)$ is gauge-equivalent to $U^{R}\left(\lambda_{R}\right)$ under the identification $(4.5)$.

It would be interesting to see the gauge-equivalence at $r / s$-matrix level. The $r / s$ matrices are derived from the following Poisson brackets,

$$
\begin{aligned}
\left\{L_{x}^{L}\left(x ; \lambda_{L}\right) \stackrel{\otimes}{,} L_{x}^{L}\left(y ; \mu_{L}\right)\right\}_{\mathrm{P}}= & {\left[r^{L}\left(\lambda_{L}, \mu_{L}\right), L_{x}^{L}\left(x ; \lambda_{L}\right) \otimes 1+1 \otimes L_{x}^{L}\left(y ; \mu_{L}\right)\right] \delta(x-y) } \\
& -\left[s^{L}\left(\lambda_{L}, \mu_{L}\right), L_{x}^{L}\left(x ; \lambda_{L}\right) \otimes 1-1 \otimes L_{x}^{L}\left(y ; \mu_{L}\right)\right] \delta(x-y)
\end{aligned}
$$




$$
\begin{aligned}
& -2 s^{L}\left(\lambda_{L}, \mu_{L}\right) \partial_{x} \delta(x-y), \\
\left\{L_{x}^{R}\left(x ; \lambda_{R}\right) \stackrel{\otimes}{,} L_{x}^{R}\left(y ; \mu_{R}\right)\right\}_{\mathrm{P}}= & {\left[r^{R}\left(\lambda_{R}, \mu_{R}\right), L_{x}^{R}\left(x ; \lambda_{R}\right) \otimes 1+1 \otimes L_{x}^{R}\left(y ; \mu_{R}\right)\right] \delta(x-y) } \\
& -\left[s^{R}\left(\lambda_{R}, \mu_{R}\right), L_{x}^{R}\left(x ; \lambda_{R}\right) \otimes 1-1 \otimes L_{x}^{R}\left(y ; \mu_{R}\right)\right] \delta(x-y) \\
& -2 s^{R}\left(\lambda_{R}, \mu_{R}\right) \partial_{x} \delta(x-y) .
\end{aligned}
$$

The classical $r / s$-matrices are the following:

$$
\begin{aligned}
& r^{L}\left(\lambda_{L}, \mu_{L}\right)=\frac{h\left(\lambda_{L}\right)+h\left(\mu_{L}\right)}{2\left(\lambda_{L}-\mu_{L}\right)}\left(T^{+} \otimes T^{-}+T^{-} \otimes T^{+}+T^{3} \otimes T^{3}\right), \\
& s^{L}\left(\lambda_{L}, \mu_{L}\right)=\frac{h\left(\lambda_{L}\right)-h\left(\mu_{L}\right)}{2\left(\lambda_{L}-\mu_{L}\right)}\left(T^{+} \otimes T^{-}+T^{-} \otimes T^{+}+T^{3} \otimes T^{3}\right), \\
& r^{R}\left(\lambda_{R}, \mu_{R}\right)=\frac{h\left(\lambda_{R}\right)+h\left(\mu_{R}\right)}{2\left(\lambda_{R}-\mu_{R}\right)}\left(T^{+} \otimes T^{-}+T^{-} \otimes T^{+}+T^{3} \otimes T^{3}\right), \\
& s^{R}\left(\lambda_{R}, \mu_{R}\right)=\frac{h\left(\lambda_{R}\right)-h\left(\mu_{R}\right)}{2\left(\lambda_{R}-\mu_{R}\right)}\left(T^{+} \otimes T^{-}+T^{-} \otimes T^{+}+T^{3} \otimes T^{3}\right) .
\end{aligned}
$$

Here $h(\lambda)$ is defined as

$$
h(\lambda) \equiv \frac{\lambda^{2}}{1-\lambda^{2}}
$$

It is straightforward to compute the gauge transformation laws of $r / s$-matrices. The $r$-matrix is transformed as

$$
\begin{aligned}
{\left[r^{L}\left(\lambda_{L}, \mu_{L}\right) \delta(x-y)\right]^{g}=} & g^{-1}(x) \otimes g^{-1}(y)\left[r^{L}\left(\lambda_{L}, \mu_{L}\right) \delta(x-y)\right. \\
& -\frac{1}{2}\left\{g(x) \stackrel{\otimes}{,} L_{x}^{L}\left(y ; \mu_{L}\right)\right\}_{\mathrm{P}} g^{-1}(x) \otimes 1 \\
& \left.-\frac{1}{2}\left\{L_{x}^{L}\left(x ; \lambda_{L}\right) \stackrel{\otimes}{,} g(y)\right\}_{\mathrm{P}} 1 \otimes g^{-1}(y)\right] g(x) \otimes g(y),
\end{aligned}
$$

and the $s$-matrix is transformed as

$$
\begin{aligned}
{\left[s^{L}\left(\lambda_{L}, \mu_{L}\right) \delta(x-y)\right]^{g}=} & g^{-1}(x) \otimes g^{-1}(y)\left[s^{L}\left(\lambda_{L}, \mu_{L}\right) \delta(x-y)\right. \\
& +\frac{1}{2}\left\{g(x) \stackrel{\otimes}{,} L_{x}^{L}\left(y ; \mu_{L}\right)\right\}_{\mathrm{P}} g^{-1}(x) \otimes 1 \\
& \left.-\frac{1}{2}\left\{L_{x}^{L}\left(x ; \lambda_{L}\right) \stackrel{\otimes}{,} g(y)\right\}_{\mathrm{P}} 1 \otimes g^{-1}(y)\right] g(x) \otimes g(y) .
\end{aligned}
$$

With the Poisson brackets,

$$
\left\{g(x) \stackrel{\otimes}{,} L_{x}^{L}\left(y ; \mu_{L}\right)\right\}_{\mathrm{P}}=\frac{\mu_{L}}{\mu_{L}^{2}-1}\left(T^{+} \otimes T^{-}+T^{-} \otimes T^{+}+T^{3} \otimes T^{3}\right) g(x) \otimes 1 \delta(x-y),
$$




$$
\left\{L_{x}^{L}\left(x ; \lambda_{L}\right) \stackrel{\otimes}{,} g(y)\right\}_{\mathrm{P}}=-\frac{\lambda_{L}}{\lambda_{L}^{2}-1}\left(T^{+} \otimes T^{-}+T^{-} \otimes T^{+}+T^{3} \otimes T^{3}\right) 1 \otimes g(y) \delta(x-y) .
$$

the gauge-equivalence of $r / s$-matrices are shown as

$$
\left[r^{L}\left(\lambda_{L}, \mu_{L}\right)\right]^{g}=r^{R}\left(\lambda_{R}, \mu_{R}\right), \quad\left[s^{L}\left(\lambda_{L}, \mu_{L}\right)\right]^{g}=s^{R}\left(\lambda_{R}, \mu_{R}\right) .
$$

This equivalence still holds even after squashing the target space geometry, as we will see in the next subsection.

Finally we should emphasize the advantage of $r / s$-matrix formalism. If one uses the retarded monodromy matrix in [24], instead of the $r / s$-matrix formalism, then the gauge-equivalence does not hold.

\subsection{Gauge equivalence of monodromy matrices: $C \neq 0$}

It is a turn to consider the squashed sigma model case with $C \neq 0$. Here we will show that $U^{R}\left(\lambda_{R}\right)$ is gauge-equivalent to $U^{L_{ \pm}}\left(\lambda_{L_{ \pm}}\right)$under the relation (3.18) and the rescalings $(3.27)$ and $(3.28)$.

Let us start from rewriting the Lax pair $L_{ \pm}^{L_{+}}\left(x ; \lambda_{L_{+}}\right)$as

$$
\begin{aligned}
& L_{ \pm}^{L_{+}}\left(x ; \lambda_{L_{+}}\right) \\
= & \frac{1}{1 \pm \lambda_{L_{+}}} g\left[T^{+}(1 \mp i \sqrt{C}) J_{ \pm}^{-}+T^{-}(1 \pm i \sqrt{C}) J_{ \pm}^{+}+T^{3}(1+C) J_{ \pm}^{3}\right] g^{-1} .
\end{aligned}
$$

As in the case with $C=0$, a gauge transformation of it is evaluated as

$$
\begin{aligned}
& {\left[L_{ \pm}^{L_{+}}\left(x ; \lambda_{L_{+}}\right)\right]^{g} \equiv g^{-1} L_{ \pm}^{L_{+}}\left(x ; \lambda_{L_{+}}\right) g-g^{-1} \partial_{ \pm} g } \\
= & -J_{ \pm}+\frac{1}{1 \pm \lambda_{L_{+}}}\left[T^{+}(1 \mp i \sqrt{C}) J_{ \pm}^{-}+T^{-}(1 \pm i \sqrt{C}) J_{ \pm}^{+}+T^{3}(1+C) J_{ \pm}^{3}\right] \\
= & -\frac{ \pm \lambda_{L_{+}}}{1 \pm \lambda_{L_{+}}}\left[T^{+}\left(1+\frac{i \sqrt{C}}{\lambda_{L_{+}}}\right) J_{ \pm}^{-}+T^{-}\left(1-\frac{i \sqrt{C}}{\lambda_{L_{+}}}\right) J_{ \pm}^{+}+T^{3}\left(1 \mp \frac{C}{\lambda_{L_{+}}}\right) J_{ \pm}^{3}\right] .
\end{aligned}
$$

By using the inverse relation of (3.18),

$$
\lambda_{L_{ \pm}}=\frac{\tanh \alpha}{\tanh \lambda_{R}}
$$

the gauge transformation is further rewritten as

$$
\left[L_{ \pm}^{L_{+}}\left(x ; \lambda_{L_{+}}\right)\right]^{g}=-\frac{\sinh \alpha}{\sinh \left(\alpha \pm \lambda_{R}\right)}\left[T^{+} \mathrm{e}^{\lambda_{R}} J_{ \pm}^{-}+T^{-} \mathrm{e}^{-\lambda_{R}} J_{ \pm}^{+}+T^{3} \frac{\cosh \left(\alpha \pm \lambda_{R}\right)}{\cosh \alpha} J_{ \pm}^{3}\right] .
$$


Thus, up to the rescaling (3.27), we have shown that

$$
\left[L_{ \pm}^{L_{+}}\left(x ; \lambda_{L_{+}}\right)\right]^{g} \simeq L_{ \pm}^{R}\left(x ; \lambda_{R}\right)
$$

This relation means the gauge-equivalence of monodromy matrices,

$$
g_{\infty}^{-1} \cdot U^{L_{+}}\left(\lambda_{L_{+}}\right) \cdot g_{\infty} \simeq U^{R}\left(\lambda_{R}\right)
$$

Note that only half of the range of $\lambda_{R}$ is covered by $\lambda_{L_{+}}$, as we know from (3.19).

The same argument is possible for $U^{L_{-}}\left(\lambda_{L_{-}}\right)$. Only the difference is that the rescaling (3.28) has to be used instead of (3.27). Then we obtain that

$$
g_{\infty}^{-1} \cdot U^{L_{-}}\left(\lambda_{L_{-}}\right) \cdot g_{\infty} \simeq U^{R}\left(\lambda_{R}\right)
$$

The remaining range of $\lambda_{R}$ is covered by $\lambda_{-}$. Thus, putting (4.14) and (4.15) together, we have shown that $U^{R}\left(\lambda_{R}\right)$ is gauge-equivalent to $U^{L_{ \pm}}\left(\lambda_{L_{ \pm}}\right)$.

Let us comment on the rescalings (3.27) and (3.28). They can be expressed as a transformation generated by $\mathrm{e}^{\mp i T^{3} \lambda_{R}}$. Then the Lax pair is transformed as

$$
L_{\mu}^{R_{ \pm}}\left(x ; \lambda_{R}\right)=\mathrm{e}^{ \pm i T^{3} \lambda_{R}} L_{\mu}^{R}\left(x ; \lambda_{R}\right) \mathrm{e}^{\mp i T^{3} \lambda_{R}} .
$$

With this transformation law, the gauge-equivalence of monodromy matrices is represented by a simple form,

$$
\widetilde{g}_{ \pm}^{-1} \cdot U^{L_{ \pm}}\left(\lambda_{L_{ \pm}}\right) \cdot \widetilde{g}_{ \pm}=U^{R}\left(\lambda_{R}\right), \quad \widetilde{g}_{ \pm} \equiv g_{\infty} \cdot \mathrm{e}^{ \pm i T^{3} \lambda_{R}}
$$

Note that $\lambda_{R}$ is a complex variable and hence the transformation (4.16) is not an $S U(2)_{\mathrm{L}}$ transformation.

The next task is to check the gauge-equivalence at the $r / s$-matrix level. Recall that the left and right $r / s$-matrices are given by [18]

$$
\begin{aligned}
& r^{L_{ \pm}}\left(\lambda_{L_{ \pm}}, \mu_{L_{ \pm}}\right)=\frac{h^{L}\left(\lambda_{L_{ \pm}}\right)+h^{L}\left(\mu_{L_{ \pm}}\right)}{2\left(\lambda_{L_{ \pm}}-\mu_{L_{ \pm}}\right)}\left(T^{+} \otimes T^{-}+T^{-} \otimes T^{+}+T^{3} \otimes T^{3}\right), \\
& s^{L_{ \pm}}\left(\lambda_{L_{ \pm}}, \mu_{L_{ \pm}}\right)=\frac{h^{L}\left(\lambda_{L_{ \pm}}\right)-h^{L}\left(\mu_{L_{ \pm}}\right)}{2\left(\lambda_{L_{ \pm}}-\mu_{L_{ \pm}}\right)}\left(T^{+} \otimes T^{-}+T^{-} \otimes T^{+}+T^{3} \otimes T^{3}\right), \\
& r^{R}\left(\lambda_{R}, \mu_{R}\right)=\frac{h^{R}\left(\lambda_{R}\right)+h^{R}\left(\mu_{R}\right)}{2 \sinh \left(\lambda_{R}-\mu_{R}\right)}\left(T^{+} \otimes T^{-}+T^{-} \otimes T^{+}+\cosh \left(\lambda_{R}-\mu_{R}\right) T^{3} \otimes T^{3}\right), \\
& s^{R}\left(\lambda_{R}, \mu_{R}\right)=\frac{h^{R}\left(\lambda_{R}\right)-h^{R}\left(\mu_{R}\right)}{2 \sinh \left(\lambda_{R}-\mu_{R}\right)}\left(T^{+} \otimes T^{-}+T^{-} \otimes T^{+}+\cosh \left(\lambda_{R}-\mu_{R}\right) T^{3} \otimes T^{3}\right) .
\end{aligned}
$$


Here scalar functions $h^{L}\left(\lambda_{L}\right)$ and $h^{R}\left(\lambda_{R}\right)$ are defined as, respectively,

$$
\begin{aligned}
h^{L}\left(\lambda_{L}\right) & \equiv \frac{C+\lambda_{L}^{2}}{1-\lambda_{L}^{2}} \\
h^{R}\left(\lambda_{R}\right) & \equiv \frac{\sinh \alpha \cosh \alpha \sinh ^{2} \lambda_{R}}{\sinh \left(\alpha-\lambda_{R}\right) \sinh \left(\alpha+\lambda_{R}\right)} .
\end{aligned}
$$

Under the gauge transformation, the rational $r / s$-matrices are transformed as

$$
\begin{aligned}
& {\left[r^{L_{ \pm}}\left(\lambda_{L_{ \pm}}, \mu_{L_{ \pm}}\right) \delta(x-y)\right]^{g} } \\
&= g^{-1}(x) \otimes g^{-1}(y)\left[r^{L_{ \pm}}\left(\lambda_{L_{ \pm}}, \mu_{L_{ \pm}}\right) \delta(x-y)-\frac{1}{2}\left\{g(x) \stackrel{\otimes}{,} L_{x}^{L_{ \pm}}\left(y ; \mu_{L_{ \pm}}\right)\right\}_{\mathrm{P}} g^{-1}(x) \otimes 1\right. \\
&\left.\quad-\frac{1}{2}\left\{L_{x}^{L_{ \pm}}\left(x ; \lambda_{L_{ \pm}}\right) \stackrel{\otimes}{,} g(y)\right\}_{\mathrm{P}} 1 \otimes g^{-1}(y)\right] g(x) \otimes g(y) \\
& {\left[s^{L_{ \pm}}\left(\lambda_{L_{ \pm}}, \mu_{L_{ \pm}}\right) \delta(x-y)\right]^{g} } \\
&= g^{-1}(x) \otimes g^{-1}(y)\left[s^{L_{ \pm}}\left(\lambda_{L_{ \pm}}, \mu_{L_{ \pm}}\right) \delta(x-y)+\frac{1}{2}\left\{g(x) \stackrel{\otimes}{,} L_{x}^{L_{ \pm}}\left(y ; \mu_{L_{ \pm}}\right)\right\}_{\mathrm{P}} g^{-1}(x) \otimes 1\right. \\
&\left.\quad-\frac{1}{2}\left\{L_{x}^{L_{ \pm}}\left(x ; \lambda_{L_{ \pm}}\right) \stackrel{\otimes}{,} g(y)\right\}_{\mathrm{P}} 1 \otimes g^{-1}(y)\right] g(x) \otimes g(y) .
\end{aligned}
$$

By using the following Poisson brackets,

$$
\begin{aligned}
& \left\{g(x) \stackrel{\otimes}{,} L_{x}^{L_{ \pm}}\left(y ; \mu_{L}\right)\right\}_{\mathrm{P}} \\
= & \left\{\frac{-\mu_{L}}{1-\mu_{L}^{2}}\left(T^{+} \otimes T^{-}+T^{-} \otimes T^{+}+T^{3} \otimes T^{3}\right)\right. \\
& \left. \pm \frac{\sqrt{C}}{1-\mu_{L}^{2}}\left[T^{+} \otimes T^{-}+T^{-} \otimes T^{+}+T^{3} \otimes T^{3}, g T^{3} g^{-1}(x) \otimes 1\right]\right\} g(x) \otimes 1 \delta(x-y), \\
& \left\{L_{x}^{L_{ \pm}}\left(x ; \lambda_{L}\right) \stackrel{\otimes}{,} g(y)\right\}_{\mathrm{P}} \\
= & \left\{\frac{\lambda_{L}}{1-\lambda_{L}^{2}}\left(T^{+} \otimes T^{-}+T^{-} \otimes T^{+}+T^{3} \otimes T^{3}\right)\right. \\
& \left.\mp \frac{\sqrt{C}}{1-\lambda_{L}^{2}}\left[T^{+} \otimes T^{-}+T^{-} \otimes T^{+}+T^{3} \otimes T^{3}, 1 \otimes g T^{3} g^{-1}(y)\right]\right\} 1 \otimes g(y) \delta(x-y),
\end{aligned}
$$

and the rescalings (3.27) and (3.28), the gauge-equivalence of $r / s$-matrices are shown as

$$
\left[r^{L_{ \pm}}\left(\lambda_{L_{ \pm}}, \mu_{L_{ \pm}}\right)\right]^{g} \simeq r^{R}\left(\lambda_{R}, \mu_{R}\right), \quad\left[s^{L_{ \pm}}\left(\lambda_{L_{ \pm}}, \mu_{L_{ \pm}}\right)\right]^{g} \simeq s^{R}\left(\lambda_{R}, \mu_{R}\right)
$$


At first glance, it might seem contradictory because the number of poles of $h^{L}\left(\lambda_{L}\right)$ is two and that of $h^{R}\left(\lambda_{R}\right)$ is four. However, the map (3.19) means that the range of $\lambda_{R}$ is divided into the two regions, hence the number of poles is also compatible. This is the case for the pole of $r$-matrix apart from those in $h^{L}\left(\lambda_{L}\right)$ and $h^{R}\left(\lambda_{R}\right)$. Its number is just one and exactly agrees with the number in either of the rational descriptions.

Finally we should comment on the $C \rightarrow 0$ limit. The relation (4.12) is reduced to the relation (4.5) in the $C \rightarrow 0$ limit with the rescaling (2.9).

\subsection{Reduced trigonometric description and integrability}

In the previous argument, one may have noticed the possibility that a couple of the two Lax pairs

$$
\begin{aligned}
& L_{ \pm}^{R_{+}}\left(x ; \lambda_{R_{+}}\right) \\
= & -\frac{\sinh \alpha}{\sinh \left(\alpha \pm \lambda_{R_{+}}\right)}\left[\mathrm{e}^{-\lambda_{R_{+}}} T^{-} J_{ \pm}^{+}+\mathrm{e}^{\lambda_{R_{+}}} T^{+} J_{ \pm}^{-}+\frac{\cosh \left(\alpha \pm \lambda_{R_{+}}\right)}{\cosh \alpha} T^{3} J_{ \pm}^{3}\right], \\
& L_{ \pm}^{R_{-}}\left(x ; \lambda_{R_{-}}\right) \\
= & -\frac{\sinh \alpha}{\sinh \left(\alpha \pm \lambda_{R_{-}}\right)}\left[\mathrm{e}^{\lambda_{R_{-}}} T^{-} J_{ \pm}^{+}+\mathrm{e}^{-\lambda_{R_{-}}} T^{+} J_{ \pm}^{-}+\frac{\cosh \left(\alpha \pm \lambda_{R_{-}}\right)}{\cosh \alpha} T^{3} J_{ \pm}^{3}\right],
\end{aligned}
$$

are available in the trigonometric description, instead of the Lax pair $L_{\mu}^{R}\left(x ; \lambda_{R}\right)$ in (2.6). Now that two spectral parameters $\lambda_{R_{ \pm}}$are contained in the Lax pairs (4.22), the periodicity of $\operatorname{Im} \lambda_{R_{ \pm}}$is $\pi$, not $2 \pi$ :

$$
L_{\mu}^{R_{ \pm}}\left(x ; \lambda_{R_{ \pm}}+\pi i\right)=L_{\mu}^{R_{ \pm}}\left(x ; \lambda_{R_{ \pm}}\right) .
$$

This observation implies that the Lax pair (2.6) is "reducible" in some sense. In fact, it is straightforward to check that each of the Lax pairs (4.22) leads to the identical classical equations of motion (2.5). Hence it really works well as the Lax pair, at least, at the classical level, though it is unclear whether it works even at the quantum level or not.

The two spectral parameters decompose the relation (3.18) into the two relations,

$$
\lambda_{L_{ \pm}}=\frac{\tanh \alpha}{\tanh \lambda_{R_{ \pm}}}
$$

With the relation (4.24), a gauge-transformation of $L_{ \pm}^{L_{+}}\left(x ; \lambda_{L_{+}}\right)$can be shown as

$$
\left[L_{ \pm}^{L_{+}}\left(x ; \lambda_{L_{+}}\right)\right]^{g}=g^{-1} L_{ \pm}^{L_{+}}\left(x ; \lambda_{L_{+}}\right) g-g^{-1} \partial_{ \pm} g
$$




$$
\begin{aligned}
& =-\frac{ \pm \lambda_{L_{+}}}{1 \pm \lambda_{L_{+}}}\left[T^{+}\left(1+\frac{i \sqrt{C}}{\lambda_{L_{+}}}\right) J_{ \pm}^{-}+T^{-}\left(1-\frac{i \sqrt{C}}{\lambda_{L_{+}}}\right) J_{ \pm}^{+}+T^{3}\left(1 \mp \frac{C}{\lambda_{L_{+}}}\right) J_{ \pm}^{3}\right] \\
& =-\frac{\sinh \alpha}{\sinh \left(\alpha \pm \lambda_{R_{+}}\right)}\left[T^{+} \mathrm{e}^{\lambda_{R_{+}} J_{ \pm}^{-}}+T^{-} \mathrm{e}^{-\lambda_{R_{+}}} J_{ \pm}^{+}+T^{3} \frac{\cosh \left(\alpha \pm \lambda_{R_{+}}\right)}{\cosh \alpha} J_{ \pm}^{3}\right] \\
& =L_{ \pm}^{R_{+}}\left(x ; \lambda_{R_{+}}\right) \text {. }
\end{aligned}
$$

Thus we have shown the gauge-equivalence as

$$
\left[L_{ \pm}^{L_{+}}\left(x ; \lambda_{L_{+}}\right)\right]^{g}=L_{ \pm}^{R_{+}}\left(x ; \lambda_{R_{+}}\right),
$$

without the rescalings of $\operatorname{sl}(2)$ generators. The gauge-equivalence of $L_{ \pm}^{L_{-}}\left(x ; \lambda_{L_{-}}\right)$and $L_{ \pm}^{R_{-}}\left(x ; \lambda_{R_{-}}\right)$can also be shown in the same way.

To summarize, the monodromy matrices satisfy the relations,

$$
g_{\infty}^{-1} \cdot U^{L_{ \pm}}\left(\lambda_{L_{ \pm}}\right) \cdot g_{\infty}=U^{R_{ \pm}}\left(\lambda_{R_{ \pm}}\right)
$$

The next is to consider the $r / s$-matrices related to a pair of the Lax pairs (4.22). From the Poisson brackets of the spatial components of the Lax pairs (4.22), similarly, one can read off the $r / s$-matrices,

$$
\begin{aligned}
r^{R_{ \pm}}\left(\lambda_{R_{ \pm}}, \mu_{R_{ \pm}}\right)= & \frac{h^{R}\left(\lambda_{R_{ \pm}}\right)+h^{R}\left(\mu_{R_{ \pm}}\right)}{2 \sinh \left(\lambda_{R_{ \pm}}-\mu_{R_{ \pm}}\right)}\left(\mathrm{e}^{ \pm\left(\lambda_{R_{ \pm}}-\mu_{R_{ \pm}}\right)} T^{+} \otimes T^{-}\right. \\
& \left.+\mathrm{e}^{\mp\left(\lambda_{R_{ \pm}}-\mu_{R_{ \pm}}\right)} T^{-} \otimes T^{+}+\cosh \left(\lambda_{R_{ \pm}}-\mu_{R_{ \pm}}\right) T^{3} \otimes T^{3}\right), \\
s^{R_{ \pm}}\left(\lambda_{R_{ \pm}}, \mu_{R_{ \pm}}\right)= & \frac{h^{R}\left(\lambda_{R_{ \pm}}\right)-h^{R}\left(\mu_{R_{ \pm}}\right)}{2 \sinh \left(\lambda_{R_{ \pm}}-\mu_{R_{ \pm}}\right)}\left(\mathrm{e}^{ \pm\left(\lambda_{R_{ \pm}}-\mu_{R_{ \pm}}\right)} T^{+} \otimes T^{-}\right. \\
& \left.+\mathrm{e}^{\mp\left(\lambda_{R_{ \pm}}-\mu_{R_{ \pm}}\right)} T^{-} \otimes T^{+}+\cosh \left(\lambda_{R_{ \pm}}-\mu_{R_{ \pm}}\right) T^{3} \otimes T^{3}\right) .
\end{aligned}
$$

Here a scalar function $h^{R}\left(\lambda_{R_{ \pm}}\right)$is already introduced in (4.19). The $r / s$-matrices satisfy the extended Yang-Baxter equation (2.16), and the classical integrability has also been shown based on the Lax pair (4.22).

Note that the range of $\lambda_{R_{ \pm}}$is restricted to half of the original trigonometric one as in (4.23). So the number of poles in $h^{R}\left(\lambda_{R_{ \pm}}\right)$is just two and it agrees with that in either of the rational descriptions. This is the case for the poles of the $r$-matrix apart from the poles in $h^{R}\left(\lambda_{R_{ \pm}}\right)$and it is just one. Thus the $r$-matrix is really of rational type in the sense of [25], though it does not look so. 
This can be confirmed by showing that the $r / s$-matrices in the rational and reduced trigonometric descriptions are related each other by a gauge transformation. The Poisson brackets (4.20) and (4.21) lead to the transformation laws

$$
\begin{aligned}
& {\left[r^{L_{ \pm}}\left(\lambda_{L_{ \pm}}, \mu_{L_{ \pm}}\right) \delta(x-y)\right]^{g}=r^{R_{ \pm}}\left(\lambda_{R_{ \pm}}, \mu_{R_{ \pm}}\right) \delta(x-y)} \\
& {\left[s^{L_{ \pm}}\left(\lambda_{L_{ \pm}}, \mu_{L_{ \pm}}\right) \delta(x-y)\right]^{g}=s^{R_{ \pm}}\left(\lambda_{R_{ \pm}}, \mu_{R_{ \pm}}\right) \delta(x-y)}
\end{aligned}
$$

without rescaling the $s l(2)$ generators. The relations (4.30) and (4.31) confirm that the $r / s$-matrices (4.28) and (4.29) should be regarded as those of rational type.

Thus the trigonometric Lax pair (2.6) is really reducible to a pair of the rational Lax pairs (4.22) applicable to the classical analysis of the squashed sigma models. It would be interesting to consider how to interpret the reducibility of the Lax pair (2.6), at the quantum level, especially in the language of Bethe ansatz [22, 34-37].

\section{Conclusion and discussion}

We have shown the gauge-equivalence of monodromy matrices in the trigonometric and rational descriptions under the relation of spectral parameters and the rescalings of $\operatorname{sl}(2)$ generators. As a result, the trigonometric description has been shown to be equivalent to a pair of the rational descriptions. That is, the "global" equivalence is accurately realized even after squashing the target space geometry. Moreover, we have found the trigonometric description is reducible to a pair of the "reduced" trigonometric descriptions, each of which is of rational class and works well as the Lax pair at the classical level. With this description, the equivalence of monodromy matrices has become very apparent.

The equivalence implies that a squashed sphere is represented by a pair of round spheres as a dipole from the viewpoint of classical integrability. This is equivalent to say that a warped $\mathrm{AdS}_{3}$ space is a pair of undeformed $\mathrm{AdS}_{3}$ spaces via a double Wick rotation. This dipole-like structure of target space would correspond to that of dipole $\mathrm{CFT}_{2}$ in warped $\mathrm{AdS}_{3} / \mathrm{CFT}_{2}[12,13]$. It is a challenging issue to try to establish the correspondence in the scenario.

In this direction the rational description would play an important role based on the "global" equivalence because a Virasoro symmetry is realized as a reprametrization of the initial data in the solution generating techniques, as dicussed in [38-40]. This Virasoro symmetry is different from the one coming from the classical conformal sym-

metry of the system. Thus we speculate that the former Virasoro algebra and the initial 
data can be related to the quantities in the conjectured dual "dipole CFT" $[12,13]$. This scenario might give a successful way to identify the dual CFT at the sigma model level, while the asymptotic symmetry analysis at the gravity level has not completely succeeded so far. Similarly, the related Kac-Moody algebra can also be discussed [38$40]^{8}$. It would also be interesting to seek a direct connection to the theorem recently presented by Hofman and Strominger [42].

It is of importance to look for the purely mathematical formulation of the correspondence between a quantum affine algebra and a pair of Yangians, without the sigma model framework. Another issue is to consider the RTT relation in light of the correspondence. It would be useful to follow the quantum treatment of quantum affine algebra [43] and the Bethe ansatz [34-37]. Notably, the trigonometric and rational S-matrices are contained in the Bethe ansatz. If the equivalence shown here survives quantization, the Bethe ansatz may be rewritten into the one consisting of only the rational S-matrices but with the same spectrum.

It would also be nice to consider the similar correspondence of monodromy matrices in the case of null-warped $\mathrm{AdS}_{3}$, where the broken $S L(2)_{\mathrm{R}}$ symmetry is realized as a $q$-deformed Poincare symmetry [44]. Its affine extension has not been clarified yet, but, conversely, it may be done by using the gauge-equivalence of monodromy matrices.

\section{Acknowledgments}

We would like to thank S. Moriyama for useful discussions. The work of IK was supported by the Japan Society for the Promotion of Science (JSPS). The work of KY was supported by the scientific grants from the Ministry of Education, Culture, Sports, Science and Technology (MEXT) of Japan (No.22740160). This work was also supported in part by the Grant-in-Aid for the Global COE Program "The Next Generation of Physics, Spun from Universality and Emergence" from MEXT, Japan. One of the author TM also would like to thank A. Molev for valuable discussions and comments.

\section{References}

[1] J. M. Maldacena, "The large N limit of superconformal field theories and supergravity," Adv. Theor. Math. Phys. 2 (1998) 231 [Int. J. Theor. Phys. 38 (1999) 1113]. [arXiv:hep-th/9711200].

[2] N. Beisert et al., "Review of AdS/CFT Integrability: An Overview," arXiv:1012.3982 [hep-th].

\footnotetext{
${ }^{8}$ It would also be nice to consider the relation to the scenario discussed for KdV equations [41].
} 
[3] R. G. Leigh and M. J. Strassler, "Exactly marginal operators and duality in four-dimensional N=1 supersymmetric gauge theory," Nucl. Phys. B 447 (1995) 95 [arXiv:hep-th/9503121].

[4] O. Lunin and J. M. Maldacena, "Deforming field theories with $U(1) \times U(1)$ global symmetry and their gravity duals," JHEP 0505 (2005) 033 [arXiv:hep-th/0502086].

[5] S. Frolov, "Lax pair for strings in Lunin-Maldacena background," JHEP 0505 (2005) 069 [arXiv:hep-th/0503201].

[6] D. Berenstein and D. H. Correa, "Emergent geometry from $q$-deformations of $\mathrm{N}=4$ super Yang-Mills," JHEP 0608 (2006) 006 [arXiv:hep-th/0511104].

[7] N. Beisert and P. Koroteev, "Quantum Deformations of the One-Dimensional Hubbard Model," J. Phys. A A 41 (2008) 255204 [arXiv:0802.0777 [hep-th]].

[8] N. Beisert, W. Galleas and T. Matsumoto, "A Quantum Affine Algebra for the Deformed Hubbard Chain," arXiv:1102.5700 [math-ph].

[9] B. Hoare, T. J. Hollowood and J. L. Miramontes, "q-Deformation of the $A d S_{5} x S^{5}$ Superstring S-matrix and its Relativistic Limit," arXiv:1112.4485 [hep-th].

[10] E. D'Hoker and P. Kraus, "Charged magnetic brane solutions in $\mathrm{AdS}_{5}$ and the fate of the third law of thermodynamics," JHEP 1003 (2010) 095;

[arXiv:0911.4518 [hep-th]]; "Holographic metamagnetism, quantum criticality, and crossover behavior," JHEP 1005 (2010) 083. [arXiv:1003.1302 [hep-th]].

[11] M. Guica, T. Hartman, W. Song and A. Strominger, "The Kerr/CFT correspondence," Phys. Rev. D 80 (2009) 124008. [arXiv:0809.4266 [hep-th]].

[12] S. El-Showk and M. Guica, "Kerr/CFT, dipole theories and nonrelativistic CFTs," arXiv:1108.6091 [hep-th].

[13] W. Song and A. Strominger, "Warped $\mathrm{AdS}_{3} /$ Dipole-CFT Duality," arXiv:1109.0544 [hep-th].

[14] I. Kawaguchi and K. Yoshida, "Hidden Yangian symmetry in sigma model on squashed sphere," JHEP 1011 (2010) 032. [arXiv:1008.0776 [hep-th]].

[15] I. Kawaguchi, D. Orlando and K. Yoshida, "Yangian symmetry in deformed WZNW models on squashed spheres," Phys. Lett. B 701 (2011) 475. [arXiv:1104.0738 [hep-th]].

[16] I. Kawaguchi and K. Yoshida, "Hybrid classical integrability in squashed sigma models," Phys. Lett. B 705 (2011) 251 [arXiv:1107.3662 [hep-th]].

[17] I. Kawaguchi, T. Matsumoto and K. Yoshida, "The classical origin of quantum affine algebra in squashed sigma models," arXiv:1201.3058 [hep-th].

[18] I. Kawaguchi and K. Yoshida, "Hybrid classical integrable structure of squashed sigma models: A short summary,” J. Phys. Conf. Ser. 343 (2012) 012055 
[arXiv:1110.6748 [hep-th]].

[19] D. Orlando, S. Reffert and L. I. Uruchurtu, "Classical integrability of the squashed three-sphere, warped AdS3 and Schrödinger spacetime via T-Duality," J. Phys. A 44 (2011) 115401. [arXiv:1011.1771 [hep-th]].

[20] P. Forgacs, "A 2-D Integrable axion model and target space duality," hep-th/0111124.

[21] I. V. Cherednik, "Relativistically Invariant Quasiclassical Limits Of Integrable Two-Dimensional Quantum Models," Theor. Math. Phys. 47 (1981) 422 [Teor. Mat. Fiz. 47 (1981) 225].

[22] L. D. Faddeev and N. Y. Reshetikhin, "Integrability of the principal chiral field model in (1+1)-dimension," Annals Phys. 167 (1986) 227.

[23] J. M. Maillet, "New integrable canonical structures in two-dimensional models," Nucl. Phys. B 269 (1986) 54.

[24] A. Duncan, H. Nicolai and M. Niedermaier, "On the Poisson bracket algebra of monodromy matrices," Z. Phys. C 46 (1990) 147.

[25] A. A. Belavin and V. G. Drinfeld, "Solutions of the classical Yang-Baxter equations for simple Lie algebras," Funct. Anal. Appl. 16 (1982) 159.

[26] V. G. Drinfel'd, "Hopf algebras and the quantum Yang-Baxter equation," Sov. Math. Dokl. 32 (1985) 254; "Quantum groups," J. Sov. Math. 41 (1988) 898 [Zap. Nauchn. Semin. 155, 18 (1986)]; "A new realization of Yangians and quantized affine algebras," Sov. Math. Dokl. 36 (1988) 212.

[27] M. Jimbo, "A $q$ difference analog of $U(g)$ and the Yang-Baxter equation," Lett. Math. Phys. 10 (1985) 63.

[28] M. Lüscher, "Quantum nonlocal charges and absence of particle production in the two-dimensional nonlinear sigma model," Nucl. Phys. B 135 (1978) 1.

[29] M. Lüscher and K. Pohlmeyer, "Scattering of massless lumps and nonlocal charges in the two-dimensional classical nonlinear sigma model," Nucl. Phys. B 137 (1978) 46.

[30] E. Brezin, C. Itzykson, J. Zinn-Justin and J. B. Zuber, "Remarks about the existence of nonlocal charges in two-dimensional models," Phys. Lett. B 82 (1979) 442.

[31] D. Bernard, "Hidden Yangians in 2-D massive current algebras," Commun. Math. Phys. 137 (1991) 191.

[32] N. J. MacKay, "On the classical origins of Yangian symmetry in integrable field theory," Phys. Lett. B 281 (1992) 90 [Erratum-ibid. B 308 (1993) 444].

[33] E. Abdalla, M. C. Abdalla and K. Rothe, "Non-perturbative methods in two-dimensional quantum field theory," World Scientific, 1991. 
[34] A. Polyakov and P. B. Wiegmann, "Theory of non-abelian Goldstone bosons in two dimensions," Phys. Lett. B 131 (1983) 121.

[35] P. B. Wiegmann, "Exact solution of the $\mathrm{O}(3)$ nonlinear sigma model," Phys. Lett. B 152 (1985) 209.

[36] V. A. Fateev, "The sigma model (dual) representation for a two-parameter family of integrable quantum field theories," Nucl. Phys. B 473 (1996) 509.

[37] J. Balog and P. Forgacs, "Thermodynamical Bethe ansatz analysis in an $S U(2) \times U(1)$ symmetric sigma model," Nucl. Phys. B 570 (2000) 655 [arXiv:hep-th/9906007].

[38] J. H. Schwarz, "Classical symmetries of some two-dimensional models," Nucl. Phys. B 447 (1995) 137 [hep-th/9503078].

[39] C. Devchand and J. Schiff, "Hidden symmetries of the principal chiral model unveiled," Commun. Math. Phys. 190 (1998) 675 [hep-th/9611081].

[40] H. Lu, M. J. Perry, C. N. Pope and E. Sezgin, "Kac-Moody and Virasoro Symmetries of Principal Chiral Sigma Models," Nucl. Phys. B 826 (2010) 71 [arXiv:0812.2218 [hep-th]].

[41] V. V. Bazhanov, S. L. Lukyanov and A. B. Zamolodchikov, "Integrable structure of conformal field theory, quantum KdV theory and thermodynamic Bethe ansatz," Commun. Math. Phys. 177 (1996) 381 [hep-th/9412229].

[42] D. M. Hofman and A. Strominger, "Chiral Scale and Conformal Invariance in 2D Quantum Field Theory," Phys. Rev. Lett. 107 (2011) 161601 [arXiv:1107.2917 [hep-th]].

[43] D. Bernard and A. Leclair, "Quantum group symmetries and nonlocal currents in 2-D QFT," Commun. Math. Phys. 142 (1991) 99.

[44] I. Kawaguchi and K. Yoshida, "Classical integrability of Schrodinger sigma models and q-deformed Poincare symmetry," JHEP 1111 (2011) 094 [arXiv:1109.0872 [hep-th]]. 